\title{
Chromatin-directed proteomics-identified network of endogenous androgen receptor in prostate cancer cells
}

\author{
Kaisa-Mari Launonen (D) ${ }^{1}$, Ville Paakinaho (D) ${ }^{1}$, Gianluca Sigismondo ${ }^{2}$, Marjo Malinen ${ }^{3}$, Reijo Sironen ${ }^{4,5}$, Jaana M. Hartikainen (D) ${ }^{4}$, \\ Hanna Laakso ${ }^{1}$, Tapio Visakorpi ${ }^{6,7}$, Jeroen Krijgsveld ${ }^{2,8}$, Einari A. Niskanen (D) ${ }^{1}$ and Jorma J. Palvimo (D) ${ }^{1 \times}$
}

(c) The Author(s) 2021

Treatment of prostate cancer confronts resistance to androgen receptor (AR)-targeted therapies. AR-associated coregulators and chromatin proteins hold a great potential for novel therapy targets. Here, we employed a powerful chromatin-directed proteomics approach termed ChIP-SICAP to uncover the composition of chromatin protein network, the chromatome, around endogenous AR in castration resistant prostate cancer (CRPC) cells. In addition to several expected AR coregulators, the chromatome contained many nuclear proteins not previously associated with the AR. In the context of androgen signaling in CRPC cells, we further investigated the role of a known AR-associated protein, a chromatin remodeler SMARCA4 and that of SIM2, a transcription factor without a previous association with AR. To understand their role in chromatin accessibility and AR target gene expression, we integrated data from ChIP-seq, RNA-seq, ATAC-seq and functional experiments. Despite the wide co-occurrence of SMARCA4 and AR on chromatin, depletion of SMARCA4 influenced chromatin accessibility and expression of a restricted set of AR target genes, especially those involved in cell morphogenetic changes in epithelial-mesenchymal transition. The depletion also inhibited the CRPC cell growth, validating SMARCA4's functional role in CRPC cells. Although silencing of SIM2 reduced chromatin accessibility similarly, it affected the expression of a much larger group of androgen-regulated genes, including those involved in cellular responses to external stimuli and steroid hormone stimulus. The silencing also reduced proliferation of CRPC cells and tumor size in chick embryo chorioallantoic membrane assay, further emphasizing the importance of SIM2 in CRPC cells and pointing to the functional relevance of this potential prostate cancer biomarker in CRPC cells. Overall, the chromatome of AR identified in this work is an important resource for the field focusing on this important drug target.

Oncogene (2021) 40:4567-4579; https://doi.org/10.1038/s41388-021-01887-2

\section{INTRODUCTION}

Androgens and androgen receptor (AR), a hormone-activated transcription factor (TF), are key factors driving the development and progression of prostate cancer (PCa). The AR is therefore the primary molecular target for the hormone therapy of advanced PCa [1]. Androgen deprivation therapies, especially with secondgeneration antiandrogens and androgen synthesis inhibitors are initially effective. However, since patients can still progress from advanced PCa to lethal castration-resistant prostate cancer (CRPC) [2], new therapeutic targets and biomarkers are needed. One source for targets may lie in the AR-associated chromatin proteins.

Most of the currently known nuclear receptor (NR)-interacting proteins, including those of $A R$, have been identified through genetic screens, such as two-hybrid systems, co-immunoprecipitation, and peptide fragment-based in vitro methods [3-5]. Even though affinity purification-coupled to mass spectrometry (MS) $[6,7]$ has enlightened the coregulators of NRs, it has rarely been performed in conditions that represent the natural milieu of NRs. Nevertheless, by utilizing RIME (rapid immunoprecipitation MS of endogenous proteins), Paltoglou et al. [8] and Stelloo et al. [9] have identified several endogenous AR-associated proteins from cross-linked chromatin of PCa cells.

Coregulators often reside as subunits in protein complexes and participate in the regulation of transcription in multiple ways, e.g. by modulating histone modifications and chromatin structure. Mammalian BRG1- or BRM-associated chromatin remodeling complex (BAF, SWI/SNF) changes the chromatin accessibility landscapes in cancer cells. The complex is a crucial regulator of cell cycle and proliferation [10], and a driver of PCa [11], with multiple cancer-specific roles [12-14]. Moreover, a frequently occurring TMPRSS2-ERG fusion gene translocation can re-target the BAF complexes on chromatin to promote prostate oncogenesis [15]. Mutually exclusive ATPase subunits, BRG1 (SMARCA4) and BRM (SMARCA2) are the key components for the complex function. In addition, cooperation between $A R$ and DNA sequencespecific TFs, such as FOXA1, GATA2, ERG and HOXB13, is well established [16]. Especially, the pioneer TF FOXA1 can bind to closed chromatin regions to regulate their chromatin accessibility,

\footnotetext{
${ }^{1}$ Institute of Biomedicine, Faculty of Health Sciences, University of Eastern Finland, Kuopio, Finland. ${ }^{2}$ German Cancer Research Center (DKFZ), Heidelberg, Germany. ${ }^{3}$ Department of Environmental and Biological Sciences, University of Eastern Finland, Joensuu, Finland. ${ }^{4}$ Institute of Clinical Medicine, Clinical Pathology and Forensic Medicine, University of Eastern Finland, Kuopio, Finland. ${ }^{5}$ Department of Clinical Pathology, Kuopio University Hospital, Kuopio, Finland. ${ }^{6}$ Faculty of Medicine and Health Technology, Tampere University and Tays Cancer Centre, Tampere University Hospital, Tampere, Finland. ${ }^{7}$ Fimlab Laboratories, Tampere, Finland. ${ }^{8}$ Heidelberg University, Medical Faculty, Heidelberg, Germany.

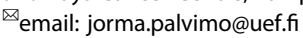



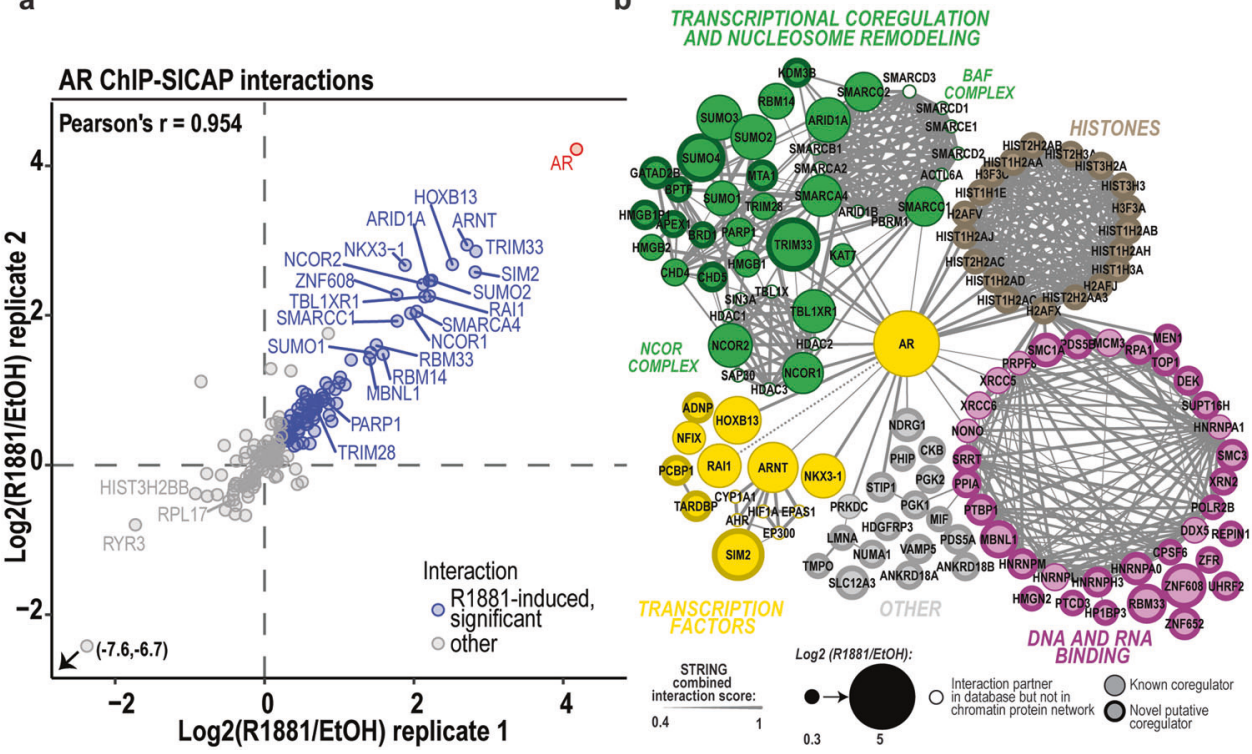

Fig. 1 ChIP-SICAP-revealed chromatome of endogenous AR in VCaP prostate cancer cells. a Scatter plot showing chromatin-associated proteins identified with AR ChIP-SICAP in two biological replicates in VCaP cells. Significantly R1881-induced (adj. $p$ value $<0.05$ ) members of AR chromatome are shown in blue and the AR highlighted in red. Gray dots represent interactions that are not R1881-induced. b Members in chromatome of AR grouped and colored by their GO: molecular function annotation. BAF and NCOR complex composition were acquired from CORUM-database and complex members that are not in the chromatome are shown with white filled nodes. Node size represents R1881-dependency (i.e. Log2(R1881/EtOH)) in chromatome and thickness of the edges represents the combined interaction score in STRINGdatabase except for RAl1 whose interaction with AR, described in [23], was not in database. Members of the network not previously linked to AR signaling are highlighted with a thicker border width of the node.

being able to facilitate chromatin binding of the AR and thus contributing to PCa carcinogenesis [17, 18].

In this work, we utilized chromatin immunoprecipitation coupled with selective isolation of chromatin-associated proteins (ChIP-SICAP) [19] to capture the chromatin protein network, the chromatome (CHROMATin proteOME) of endogenous AR in VCaP cells that represent CRPC cells [20]. In addition to anticipated chromatin remodeling factors, such as SMARCA4, we identified several nuclear proteins not previously associated with the AR. Among the latter were single-minded homolog 2 (SIM2) and aryl hydrocarbon nuclear translocator (ARNT/HIF1 $\beta$ ) that together form a heterodimeric TF. SIM2 has intriguingly been reported as a biomarker of aggressive PCa [21, 22], but not previously associated as a partner of AR or with androgen signaling. In addition to identifying the chromatome of endogenous AR in the model CRPC cells, we characterized the genome-wide role of SIM2 in chromatin accessibility, AR binding and gene expression in comparison to that of SMARCA4 in the VCaP cells. Our results uncover interesting gene and pathway-selective roles for both SMARCA4 and SIM2 in the regulation of androgen signaling in CRPC cells.

\section{RESULTS}

\section{The chromatome of AR in CRPC cells}

To complement our BiolD-derived interactome of AR [23], we used ChIP-SICAP [19] to identify the composition of chromatome around the AR in its chromatin context using differential SILAClabeling in VCaP cells exposed to R1881 (a synthetic AR agonist), or vehicle (Supplementary materials, Fig. 1a, Supplementary Figure S1a). From the 190 ChIP-SICAP quantified proteins, 87 formed a R1881-induced AR chromatome, thus uncovering proteins that may functionally interact with the receptor (Fig. 1a, Supplementary Fig. S1b, Supplementary Table 1). Overlap between the ChIP-SICAP-identified AR chromatome with two RIME-based interactomes of AR from LNCaP ( 20\%) and R1-AD1 PCa cells $(\sim 7 \%)$ is shown in Supplementary Fig. S2 [8, 9].
The R1881-induced AR chromatome was categorized to different functional protein groups (Fig. 1b). DNA- and RNAbinding proteins, histones, and DNA repair- and mRNA processingrelated proteins formed majority $(\sim 50 \%)$ of the network. DNAbinding proteins with more specialized roles, e.g. coregulator roles, in transcription formed one-third of the chromatome. This group included ARID1A, SMARCA4, SMARCC1 and SMARCC2, subunits of the BAF complex, and CHD4, a subunit of NuRD complex, coregulators NCOR1, NCOR2 and TRIM28, as well as PARP1, a regulator of DNA damage repair and transcription. An intriguing fraction $(\sim 11 \%)$ of the chromatome consisted of TFs, e.g. NKX3-1 and HOXB13. Most interestingly, the ChIP-SICAP revealed a large number $(>70 \%)$ of chromatin proteins that have not previously been identified to associate with AR or androgen signaling (Fig. $1 \mathrm{~b}$ and Supplementary Table 1). This group includes e.g. MTA1 and GATA2B, two additional subunits of the NuRD complex, BRD1, a component of the MOZ/MORF acetyltransferase complex, and CHD5, an ATP-dependent helicase. TARDBP and SIM2, two TFs and potential PCa biomarkers [21, 22, 24, 25], and prostate metastasis suppressor NDRG1 [26] also belong to the proteins not previously linked to the $A R$. From the $A R$ chromatome, we characterized and validated the roles of two proteins; SMARCA4 previously linked to the AR and SIM2 hypothesized to act as a novel pioneer TF of AR.

\section{SMARCA4 co-occupies the majority of AR-binding sites, but} has a limited effect on their chromatin accessibility

To explore the more detailed role of SMARCA4 in AR-associated chromatin environments, we first performed ChIP-seq with SMARCA4 and AR antibodies in VCaP cells in the presence or absence of 5a-dihydrotestosterone (DHT). We focused our interest on sites co-occupied by SMARCA4 and AR and how androgen affects the co-occupancy. Most chromatin-binding sites of SMARCA4 (61534) were not affected by DHT (cluster C1, Fig. 2a, Supplementary Fig. S3a). However, DHT enhances the recruitment of SMARCA4 to these sites (cluster C1, Fig. 2b). The SMARCA4- 
b

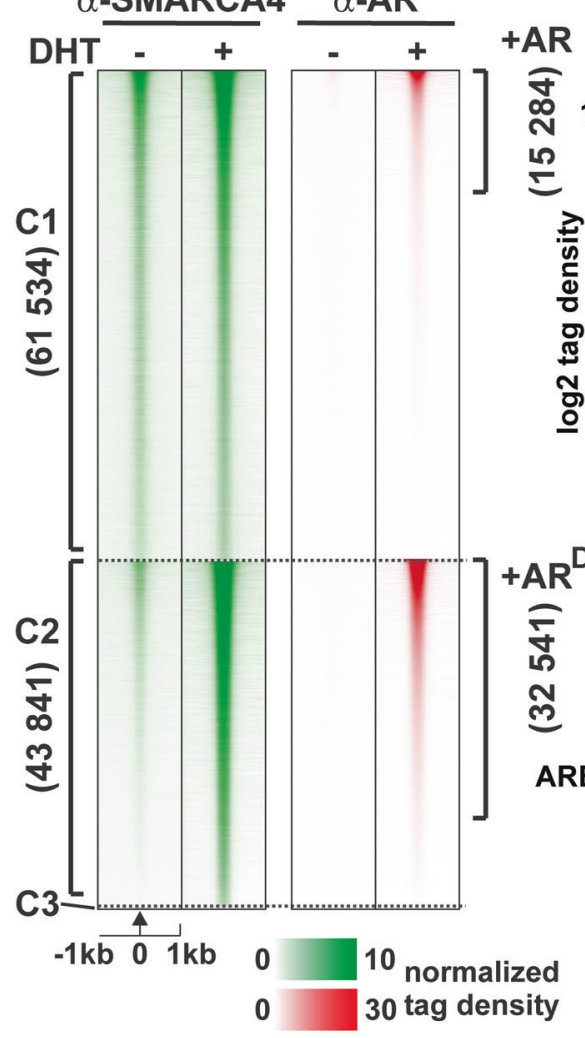

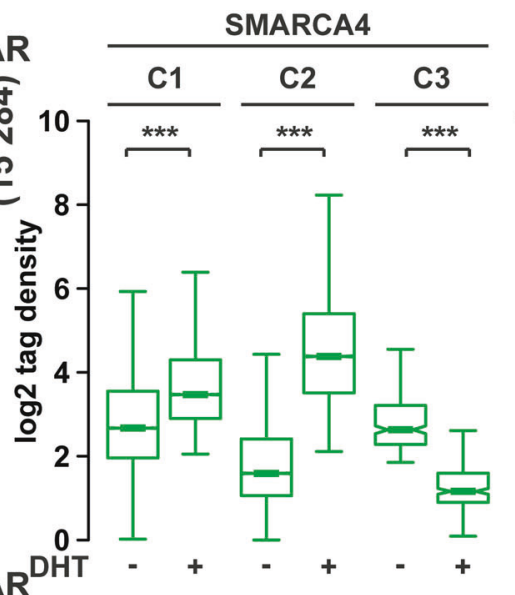

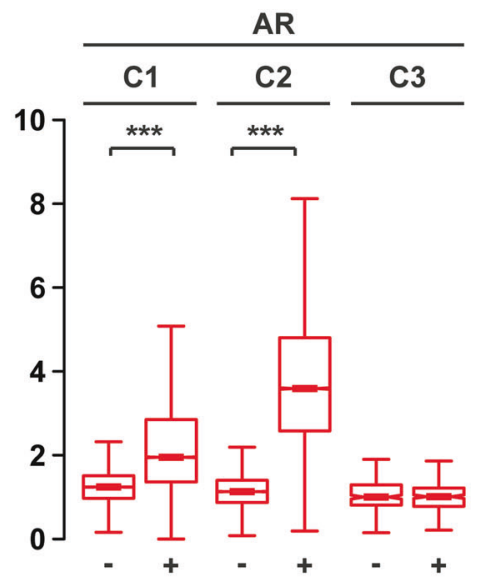

C

fold enrichment over background

0

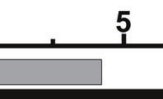

10 15 d

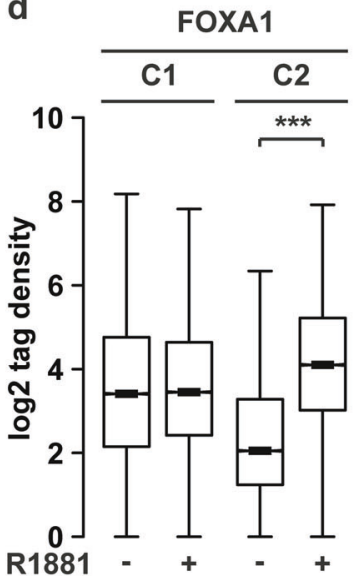

e

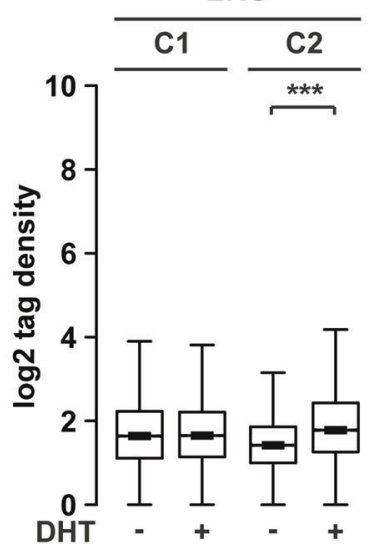

f

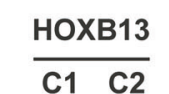

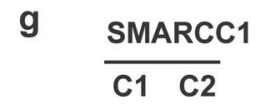

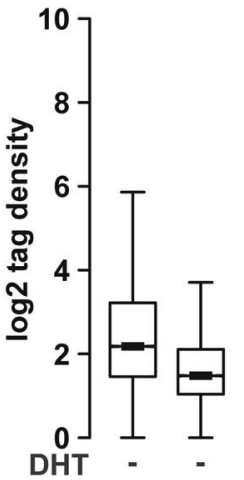

C1 $\square$

C2

Fig. 2 Activated AR recruits SMARCA4 onto chromatin in VCaP cells. a Heatmaps of normalized tag counts from ChIP-seq with SMARCA4 and AR antibodies. C1 represents cluster where SMARCA4 is recruited to chromatin independently of DHT exposure $(-3<\mathrm{FC}(\mathrm{DHT} / \mathrm{vehicle})<3)$, C2 represents DHT-induced SMARCA4 binding sites ( $F C(D H T /$ vehicle) $>3$ ) and C3 cluster where SMARCA4 binding is lost by AR activation by DHT (FC(DHT/vehicle) <-3). b Boxplots of SMARCA4 (green) and AR tag (red) counts in each cluster in panel a. c Motif analysis for two first clusters showing the fold enrichment over background. d-f Binding for FOXA1, ERG or HOXB13 at sites in clusters C1 and C2 in the presence and absence of androgen as indicated. HOXB13 data are from cells grown in normal growth media medium without added androgen. $\mathrm{g}$ SWI/ SNF complex member SMARCC1-binding in sites of clusters C1 and C2. Significant change in binding shown by asterisks $* * *<0.001$, calculated with One-way ANOVA with Bonferroni post hoc test.

binding sites (SBs) in $\mathrm{C} 1$ prevalently enrich in promoter regions (Supplementary Fig. S3b) marked with active histone modifications and active production of intergenic enhancer RNA (eRNA) without an effect of androgen (Supplementary Fig. S3b, d-f). This suggests that the $\mathrm{C} 1$ sites mostly represent VCaP cell type-specific active promoters and enhancers whose activity is mostly unresponsive to androgen. More than $40 \%$ of SBs (cluster C2) were induced upon androgen treatment and $\sim 75 \%$ of them overlapped with AR-binding sites (ARBs). At the C2 sites, AR and SMARCA4 showed a high correlation with the binding (Fig. 2a and b, cluster C2, Supplementary Fig. S3c) and they enrich in intergenic and intronic enhancer regions with intergenic eRNA production upon hormone exposure (Supplementary Fig. S3b, d-f). Only a few sites were lost upon hormone treatment (cluster C3). Examples of genome browser tracks are shown in Supplementary Fig. S3g-h. 
The motif analyses revealed that the $C 2$ sites have a higher enrichment of androgen response elements (AREs) compared with the $C 1$ sites, further supporting androgen-induced recruitment of SMARCA4 onto the chromatin. Motifs for FOXA1, ERG and HOXB13 were in turn equally enriched at the $\mathrm{C} 1$ and $\mathrm{C} 2$ sites (Fig. 2C, Supplementary Table 2). However, ChIP-seq datasets [27-29] indicated that binding of FOXA1 and ERG increased upon hormone exposure at $\mathrm{C} 2$ sites, but not at $\mathrm{C} 1$ sites (Fig. $2 \mathrm{~d}$ and e). HOXB13, however, seems to bind more at $\mathrm{C} 1$ sites (Fig. 2f). Similar to SMARCA4, SMARCC1 [15], (found in the ChIP-SICAP), shows binding onto $C 1$ and $C 2$ sites (Fig. $2 g$, Supplementary Fig. S3d), suggesting that entire BAF-complex is recruited to ARBs.

To gain more insight into how SMARCA4 influences chromatin accessibility at ARBs, we depleted SMARCA4 from VCaP cells (Supplementary Fig. S4) and performed ATAC-seq in the presence and absence of DHT. Interestingly, ATAC-seq analysis revealed that SMARCA4 depletion decreased chromatin accessibility at 8966 sites, while it increased the accessibility at 2038 sites (Supplementary Fig. S5a, b). Motifs for CTCF, FOXA1 and HOXB13 were the most enriched ones at the chromatin sites whose accessibility was decreased by SMARCA4 depletion (Supplementary Fig. S5c). ChIP-seq datasets indicated that FOXA1 and HOXB13 bind more prevalently at the sites whose accessibility was decreased by SMARCA4 depletion than the ones unaffected by the depletion (Supplementary Fig. S5d). These data suggest that SMARCA4 affect the function of FOXA1 and HOXB13.

We next focused our analyses on the changes of chromatin accessibility at the ARBs, which revealed that androgen generally increased their accessibility (Fig. 3a, ATAC, siCTRL). Despite a large overlap between SBs and ARBs, chromatin accessibility only at 2149 ARBs was reduced by SMARCA4 depletion (siSMARCA4 in Fig. $3 a$ and $b$ and examples of genome browser tracks in Supplementary Fig. S6a, b). Half of these siSMARCA4-affected sites were open regardless of the hormone exposure (pre-accessible sites) and the rest of them showed an increase in their accessibility after the hormone (de novo sites, Fig. $3 \mathrm{a}$ and $\mathrm{c}$ ). These results suggest that the chromatin remodeling by SMARCA4 at ARBs takes place both at androgen-dependent and independent enhancers.

On the pre-accessible sites, SMARCA4 depletion decreased accessibility independently of DHT, whereas at the de novo sites, the decrease was significant only with DHT. The latter result suggests that the SMARCA4 recruited by the AR increases chromatin accessibility, potentially assisting recruitment of other factors to these sites. This notion was supported by motif analyses (Fig. 3d, Supplementary Table 2). As the binding of especially FOXA1 is androgen-induced at siSMARCA4-affected sites (Fig. 3e), the ARinduced recruitment of SMARCA4 could contribute to the androgeninduced binding of FOXA1 [30]. These results indicate that the SMARCA4 has both AR-dependent and -independent roles in the regulation of chromatin landscape in CRPC cells.

\section{SMARCA4 modulates the expression of AR target genes involved in extracellular matrix organization and cell adhesion}

We next studied the genome-wide effects of SMARCA4 depletion on gene expression in VCaP cells with and without DHT using RNA-seq. Principal component analysis (PCA) showed only small differences upon SMARCA4 depletion, but the effect of DHT remained (Supplementary Fig. S7a). SMARCA4 depletion altered the expression of 1646 genes. As analyzed by Metascape [31], genes that enriched in ribosome biogenesis and translation were the top pathways downregulated by siSMARCA4 with vehicle and $\mathrm{DHT}$, respectively. Mitophagy was in turn the top pathway upregulated by siSMARCA4 with vehicle, whereas the genes upregulated by siSMARCA4 with DHT did not significantly enrich in Metascape analysis (Supplementary Fig. S7b, c). We next focused on androgen-regulated transcriptome; SMARCA4 depletion brought 1117 new genes under androgen regulation, while
480 genes lost their androgen regulation. The majority ( 70\%) of genes in the latter group were androgen (A) downregulated genes, whereas within the former group, the amount of both upregulated and downregulated genes increased approximately equally (Fig. 4a, Supplementary Fig. S7d). siSMARCA4 resulted in 931 differentially expressed genes (DEGs) compared to siCTRL with DHT, of which 363 genes were significantly androgenregulated (Fig. 4b, c, Supplementary Fig. S7e). Notably, the expression of $68 \%$ of the DEGs that are not regulated by androgen decreased upon SMARCA4 depletion (Supplementary Fig. S7e), clearly indicating that the regulatory role of SMARCA4 in CRPC cells is not restricted to androgen-regulated genes.

Differentially androgen-regulated gene sets were then subjected to Metascape analysis. SMARCA4 depletion increased the expression of androgen-responsive genes enriched in e.g., morphogenesis of a branching structure and cell morphogenesis involved in differentiation, whereas the depletion inhibited the expression of those enriched in purine metabolism and cellular response to a drug (Fig. 4d, see also Supplementary Table 3). From siSMARCA4 DEGs, only A_up/siSMARCA4_dn gene set was associated with siSMARCA4-affected chromatin sites in ATAC-seq data (Supplementary Fig. S8a-c), implying that SMARCA4-mediated changes in chromatin accessibility facilitate their expression.

We next used live-cell imaging that measures cell confluency as a proxy for cell growth and spreading to test whether the effects of SMARCA4 depletion are translated into altered growth of VCaP cells (see Supplementary materials for details). We also compared the effect of SMARCA4 depletion to that of AR depletion [27] (Supplementary Fig. S9). As shown in Fig. 5, SMARCA4 depletion did not influence the cell growth in the absence of androgen, whereas in the presence of androgen, it decreased the relative cell confluency, albeit to a lesser extent than AR depletion. Depletion of SMARCA4 similarly decreased the relative confluency of $\mathrm{LNCaP}$ cells (Supplementary Fig. S10), displaying similar expression of SMARCA4 as VCaP cells (Supplementary Fig. S11). Our results thus imply an important role for SMARCA4 in the androgen regulation of genes involved in the extracellular matrix organization and morphogenesis, pathways that include possible connections to epithelial-mesenchymal transition (EMT) in CRPC cells.

\section{Silencing of SIM2 alters chromatin accessibility at a subset of ARBs}

The SIM2, identified here as a novel TF in the AR chromatome, has been reported as a biomarker of PCa $[22,32]$. Analysis of a PCa cohort [33] concluded that the expression of SIM2 mRNA is significantly higher in PCa than in benign prostate hyperplasia, with CRPC showing a further increasing trend in the expression (Supplementary Fig. S12). Moreover, high expression of SIM2 mRNA is associated with decreased overall patient survival in TCGA PCa data (Supplementary Fig. S13). To explore the effect of SIM2 on AR-dependent transcription, we first tested if the TF could modulate the activity of AR-dependent reporter gene in VCaP cells (Supplementary Methods). Like SMARCA4, overexpression of SIM2 or its heterodimerization partner ARNT alone did not affect the reporter activity, but increased reporter activity was detected when SIM2 and ARNT were co-transfected (Supplementary Fig. S14a, b), suggesting potential for the SIM2-ARNT heterodimer in the regulation of AR-dependent transcription.

As attempts to study the chromatin binding of endogenous SIM2 using ChIP-seq failed due to lack of suitable antibodies, we silenced SIM2 (silencing confirmed by RT-qPCR, Supplementary Fig. S15) and studied whether this affects accessibility of chromatin at or close to ARBs in VCaP cells. Interestingly, ATAC-seq revealed that SIM2 silencing decreased chromatin accessibility at 10514 sites, while it increased the accessibility only at 351 sites (Supplementary Fig. S16a, b). Motifs for CTCF, FOXA1 and HOXB13 were the most enriched ones at the chromatin sites whose accessibility was decreased by SIM2 silencing (Supplementary Fig.e S16c). Analysis 

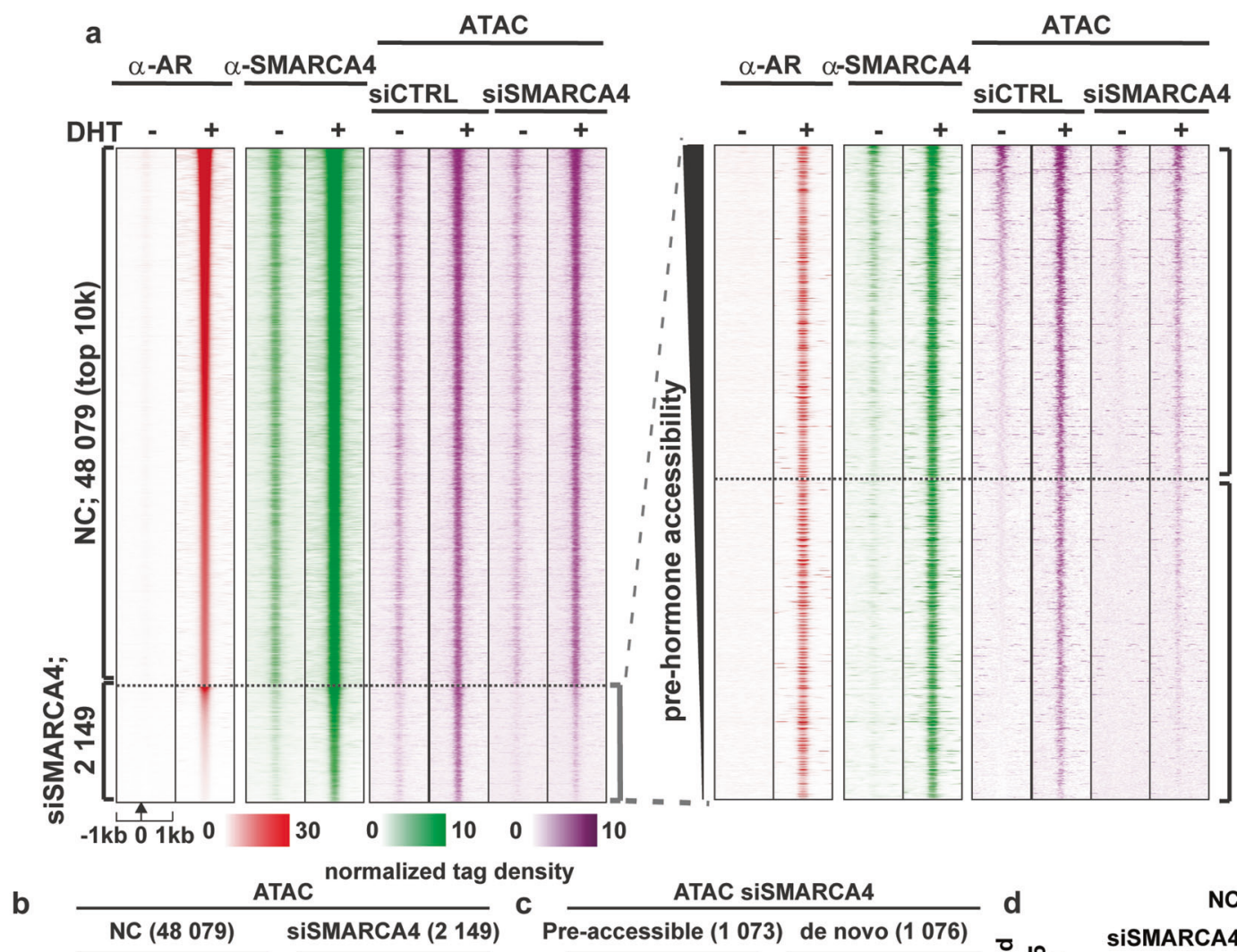

C $\frac{\text { ATAC siSMARCA4 }}{\text { Pre-accessible (1 073) de novo (1 076) }}$
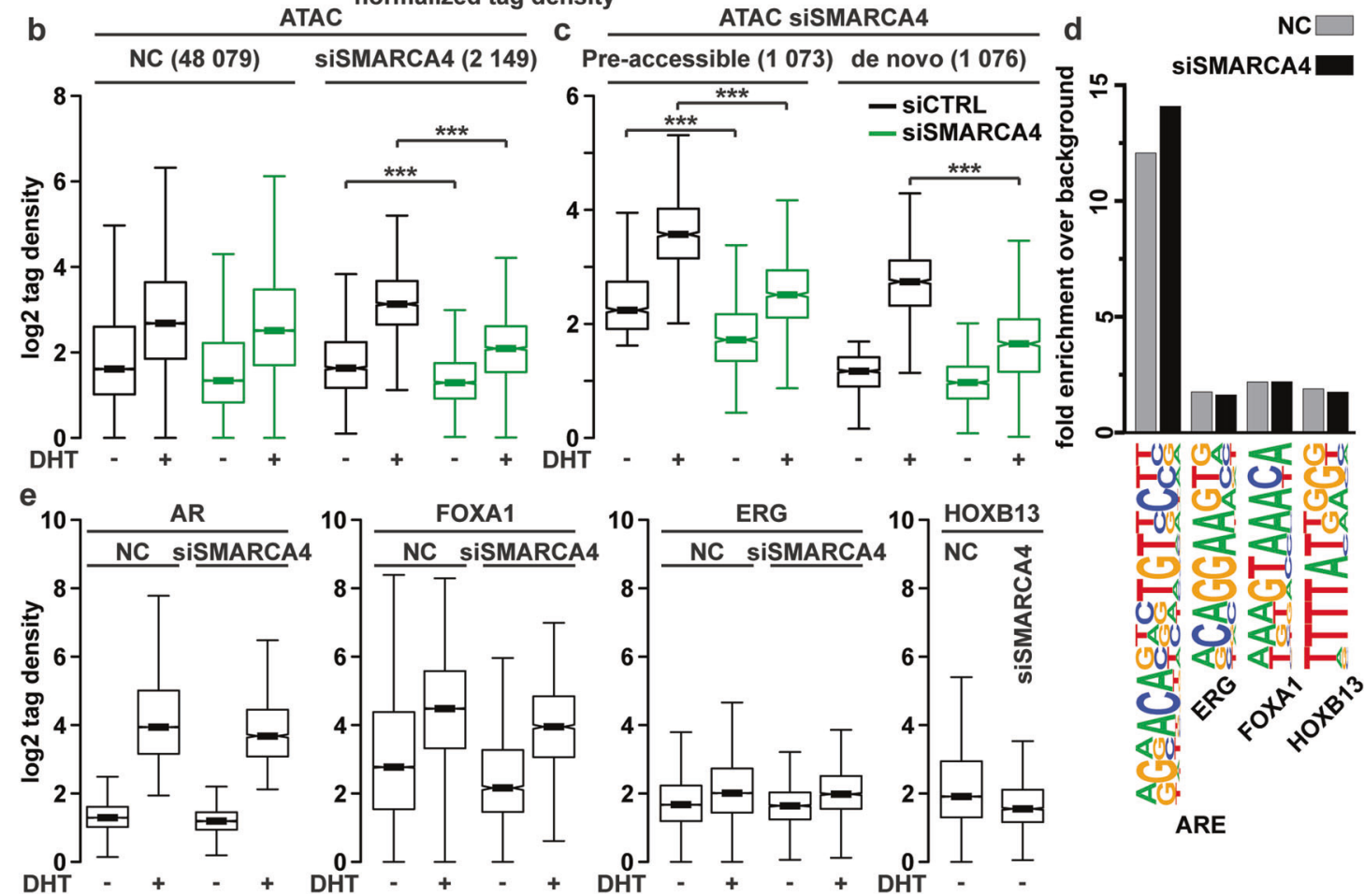

Fig. 3 Depletion of SMARCA4 decreases chromatin accessibility at a subset of AR-binding sites as assessed by ATAC-seq. a Chromatin accessibility sites as revealed by ATAC-seq in SMARCA4-depleted and control VCaP cells shown as heatmap of normalized tag counts together with SMARCA4 and AR binding. siSMARCA4-affected chromatin accessibility sites magnified and sorted by the pre-hormone (DHT) accessibility on the right. NC, sites not changed by siSMARCA4. b Boxplots of site accessibility changes by SMARCA4 depletion compared to control under vehicle or DHT exposure. c Boxplots of pre-hormone accessibility sites divided into pre-accessible sites that are open already under vehicle conditions, whereas de novo sites open under DHT exposure. $\mathbf{d}$ Motif analysis of NC and siSMARCA4-affected sites. e Binding of $A R$, FOXA1, ERG or HOXB13 on the sites in panel a in the presence and absence of androgen as indicated. Significant changes in accessibility are shown by asterisks ${ }^{* * *}<0.001$, calculated with One-way ANOVA with Bonferroni post hoc test. 


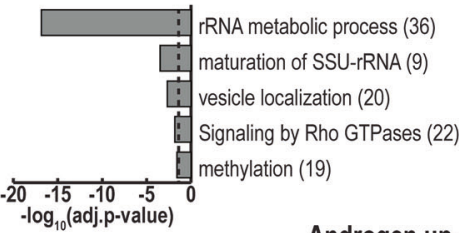

Androgen up-regulated genes
Androgen-regulated genes

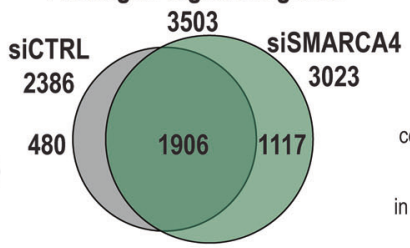

membrane trafficking (68) autophagy (53)

cell cycle $\mathrm{G} 1 / \mathrm{S}$ phase transition (35) DNA replication (32)

in utero embryonic development (39)

Androgen down-regulated genes
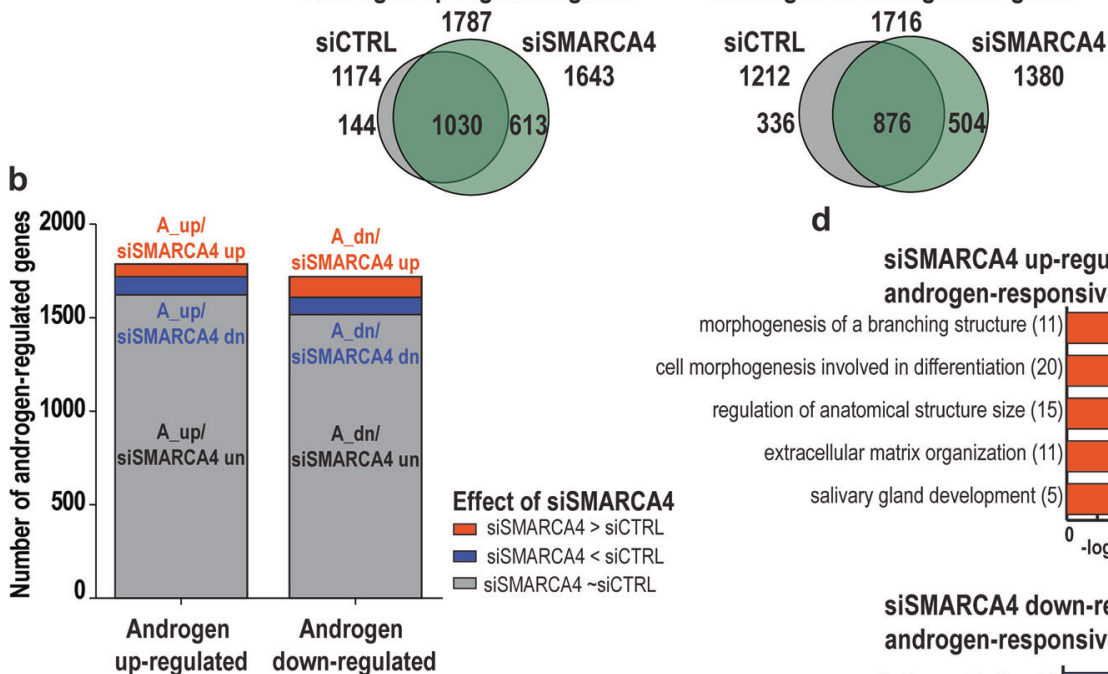

d

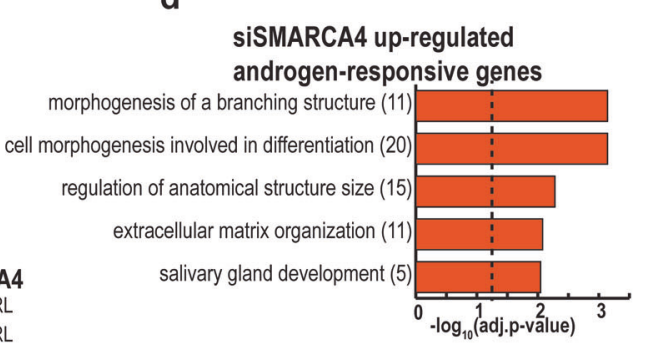

siSMARCA4 down-regulated androgen-responsive genes

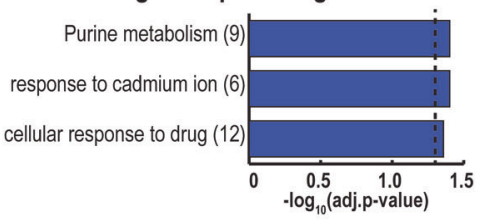

c

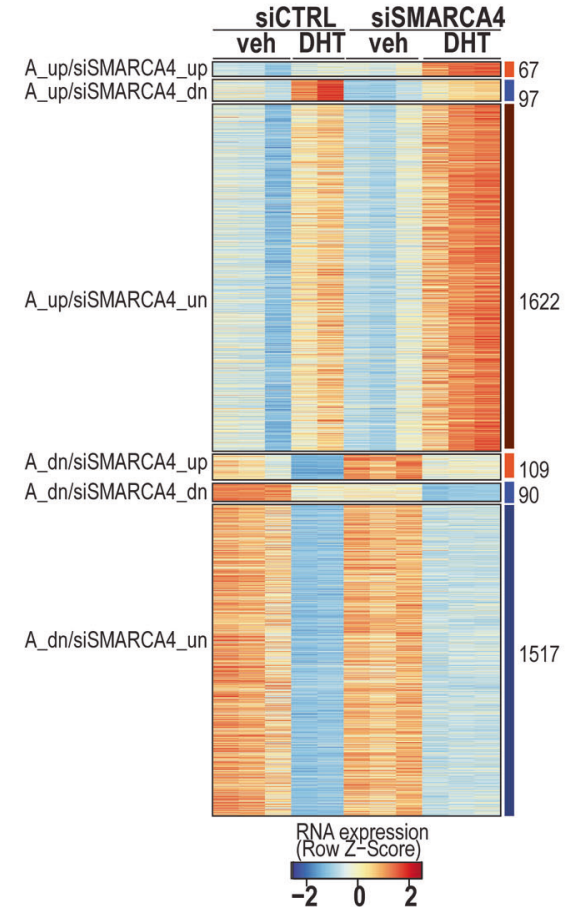

Androgen up-regulated genes not changed by siSMARCA4

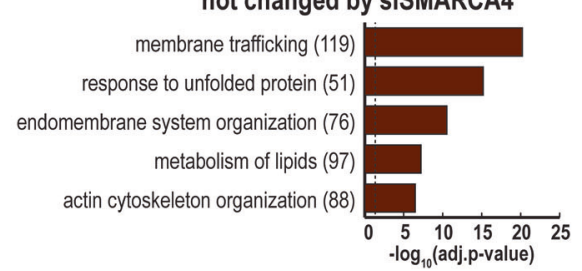

Androgen down-regulated genes not changed by siSMARCA4

rRNA metabolic process (60) regionalization $(60)$

embryonic morphogenesis (80) morphogenesis of an epithelium (74)

cellular response to lipid (80)

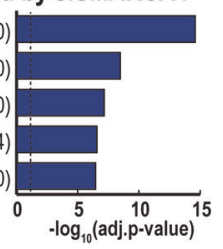

Fig. 4 Depletion of SMARCA4 in VCaP cells results in hundreds of differentially expressed androgen-regulated genes as revealed by RNAseq. a Venn-diagrams showing the overlap of androgen-regulated genes (adjusted $p$ value $<0.05$ ) in control (siCTRL) and SMARCA4-targeting siRNA (siSMARCA4) -exposed samples. The upper part represents all androgen-regulated genes, with five top enriched pathways for siCTRL unique (in gray) and siSMARCA4 unique genes (in green), and the lower part shows the subdivision of androgen-regulated genes into upregulated and downregulated subpopulations (see also Supplementary Fig. S5). b Subdivision of androgen-upregulated and -downregulated genes to six groups as defined by the effect of siSMARCA4. siSMARCA4-upregulated genes (A_up/siSMARCA4_up; A_dn/ siSMARCA4 up; adjusted $p$ value $<0.05$, log2[siSMARCA4 androgen/siCTRL androgen] $>0$ ) are shown in red, siSMARCA4-downregulated genes (A_up/siSMARCA4_dn; A_dn/siSMARCA4_dn; adjusted $p$ value $<0.05$, log2[siSMARCA4 androgen/siCTRL androgen] $<0$ ) are in blue, and unchanged genes (A_up/siSMARCA4_un; A_dn/siSMARCA4_un) are in gray. c Heatmap showing RNA expression of individual replicates ( $n=2$ for siCTRL DHT and $n=3$ for others) in the gene groups defined in b. Gene numbers belonging to each group are shown on the right. veh, vehicle. d Pathway analysis showing the five most significantly (adjusted $p$ value $<0.05$, dashed line) enriched pathways in Metascape (see Supplementary Table 3 for details) for the gene groups as defined in panel b. Number of genes associated with each biological process is shown in parenthesis. 

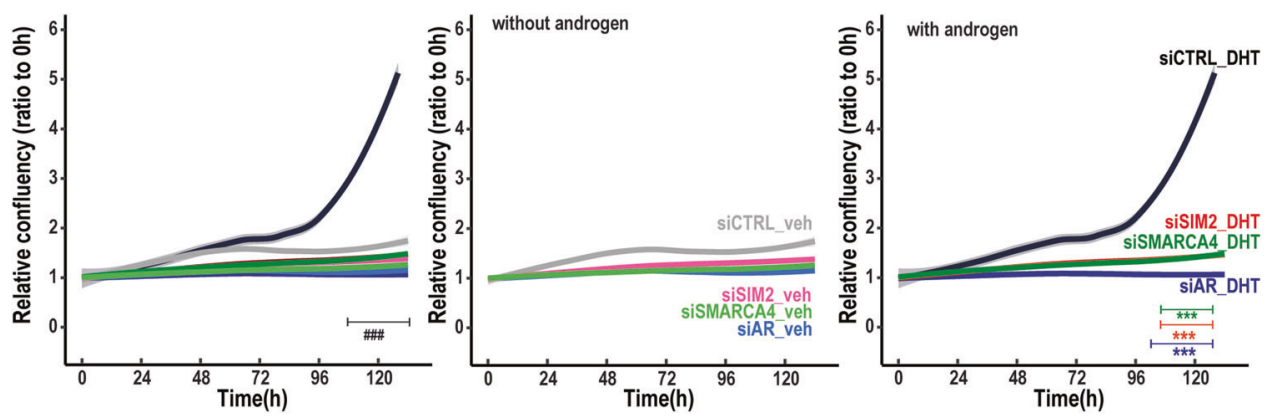

Fig. 5 Silencing of SMARCA4 and that of SIM2 attenuates proliferation of VCaP cells. Confluency of VCaP cells exposed to siCTRL, siAR, siSMARCA4 and siSIM2 as indicated was monitored by phase percentage with live cell imaging in Incucyte ${ }^{\circledR}$ in relation to the starting time point. Cells were exposed to $10 \mathrm{nM}$ DHT or vehicle (veh, ethanol) as indicated at the start of the experiment. Line presents mean and the shadow around it indicates standard deviation from four biological replicates. Significant changes to control in Two-way ANOVA and Bonferroni's multiple comparison posttests are indicated with asterisks, $p$ value ${ }^{* * *}<0.001$ and significant hormone effect with ${ }^{\# \# \#}<0.001$. On the left, all cell confluence measurements in the same graph; in the middle, measurements in the absence of androgen; on the right, measurements in the presence of DHT.

of ChIP-seq data indicated that FOXA1 and HOXB13 bind more prevalently at the sites whose accessibility was decreased by SIM2 silencing than the ones unaffected by the silencing (Supplementary Fig. S16d). These data suggest that similarly to the SMARCA4, the SIM2 might affect the function of FOXA1 and HOXB13.

We next focused our analyses on the changes of chromatin accessibility at the ARBs, which revealed that SIM2 silencing reduces the chromatin accessibility at 2434 ARBs (examples of genome browser tracks in Supplementary Fig. S17a-c). Two-thirds of the siSIM2-affected ARBs were accessible before androgen exposure (pre-accessible in Fig. $6 a-c$ ), while the rest of them became accessible after androgen exposure (de novo in Fig. 6a-c). In line with the ChIP-SICAP data, the most enriched motif at siSIM2-affected sites was the ARE, albeit it was less enriched at those sites than at the sites unaffected by siSIM2 (NC sites, Fig. 6d, Supplementary Table 2). The tag density of AR, but also that of FOXA1, ERG and HOXB13, showed an increasing trend at the siSIM2-affected sites compared with the NC sites (Fig. 6e). These results suggest that the SIM2 is a TF co-operating with $A R$ and possibly with other PCa-relevant TFs, e.g. FOXA1 or HOXB13.

To test whether SIM2 also affects the binding of AR to chromatin, we performed AR ChIP-seq after SIM2 silencing. As shown in Fig. 7, binding of the receptor to majority of ARBs was not changed. However, the SIM2 silencing affected 2265 ARBs by reducing and increasing chromatin occupancy at approximately equal number of sites (Fig. 7a, b). When reflecting these to the siSIM2-affected changes in chromatin accessibility, interestingly, a decrease in the accessibility was seen at 690 siSIM2-DN ARBs (Fig. 7c, e, f), whereas at siSIM2-UP ARBs, changes in the chromatin accessibility were not visible (Fig. 7c). The remaining (1744) siSIM2affected sites showed no change in AR binding. Thus, SIM2 might display some pioneer factor activity with the AR in certain chromatin environments, but its pioneering activity is weaker than that of FOXA1 (Supplementary Fig. S18a-d). Moreover, the majority of ARBs altered by SIM2 silencing did not overlap with ARBs altered by FOXA1 depletion (Supplementary Fig. S18e), which is supported by the motif analyses showing less enrichment of FOXA1 motif at the siSIM2-DN ARBs than at sites showing no change in AR binding (Fig. 7d, Supplementary Table 2). These results together suggest that the SIM2 can render a subset of chromatin sites more accessible to the AR.

\section{SIM2 has a marked effect on the AR-mediated gene expression}

Next, we explored the effect of SIM2 on gene expression using RNAseq after SIM2 silencing. In PCA, RNA-seq samples formed separate groups, both by siCTRL/siSIM2 and vehicle/DHT treatment (Supplementary Fig. S19a). In comparison to the SMARCA4 depletion,
SIM2 silencing had a stronger general effect on gene expression, as the expression of almost 7300 genes was altered (Supplementary Fig. S19b, c). For example, the expression of BRCA1, BRCA2 and ATM, genes involved in DNA damage repair, was increased by SIM2 silencing, whereas that of NSE, SYP and GHGA, marker genes of the neuroendocrine $\mathrm{PCa}$, was decreased by the silencing (Supplementary Fig. S19d, e). Metascape showed that cell cycle checkpoints and cell division were the top pathways upregulated by siSIM2 with vehicle and with DHT, respectively (Supplementary Fig. S19b, c, Supplementary Table 3). Interestingly, SIM2 silencing more than doubled the number of DHT-regulated genes, bringing 2394 genes under androgen regulation, while abolishing androgen regulation of only 180 genes (Fig. 8a, b). Furthermore, SIM2 silencing resulted in 6867 DEGs of which 2937 ones were androgen-regulated (Fig. 8c, d, Supplementary Fig. S19f). SIM2 silencing also alleviated the repression of AR expression by androgen (Supplementary Fig. S20). ARNT silencing essentially recapitulated the effect of SIM2 on selected AR target genes and DEGs as assessed by RT-qPCR (Supplementary Fig. S21a, b).

Metascape analysis of androgen-regulated gene sets showed a significant enrichment of several pathways by SIM2 silencing (Supplementary Table 3). The top pathways enriched in the A_up/ siSIM2_up gene set and that in the A_up/siSIM2_dn gene set were membrane trafficking and cellular responses to external stimuli, respectively (Fig. 8e, two upper graphs). On the other hand, among the androgen-downregulated genes, siSIM2 enhanced cellular response to steroid hormone stimulus and attenuated ribosome biogenesis (top pathways in A_dn/siSIM2_up and A_dn/ siSIM2_dn, respectively, Fig. 8e two lower graphs). In line with the above interpretation of the ATAC-seq data, epigenetic Landscape In Silico deletion Analysis [34] revealed enrichment of FOXA1 with the DEGs, especially among the siSIM2-upregulated genes (see Supplementary Table 4).

From the DEGs, siSIM2-upregulated genes (A_up/siSIM2_up and A_dn/siSIM2_up) were associated with siSIM2-affected sites in ATAC-seq data (Supplementary Fig. S22a), as exemplified by CA13 locus (Supplementary Fig. S22b) and UGT8 locus (Supplementary Fig. S22C). To summarize these genome-wide results, SIM2 affects chromatin accessibility at ARBs, albeit to a markedly smaller extent than FOXA1. In comparison to SMARCA4, the relatively small effect of SIM2 on chromatin accessibility of ARBs is translated to a more pronounced impact on the regulation of gene expression, affecting interesting regulatory pathways.

Proliferation of CRPC cells and their tumor size are repressed by SIM2 silencing

Since pathway analysis predicted that cell cycle checkpoints and division are affected by SIM2 silencing, we hypothesized that 

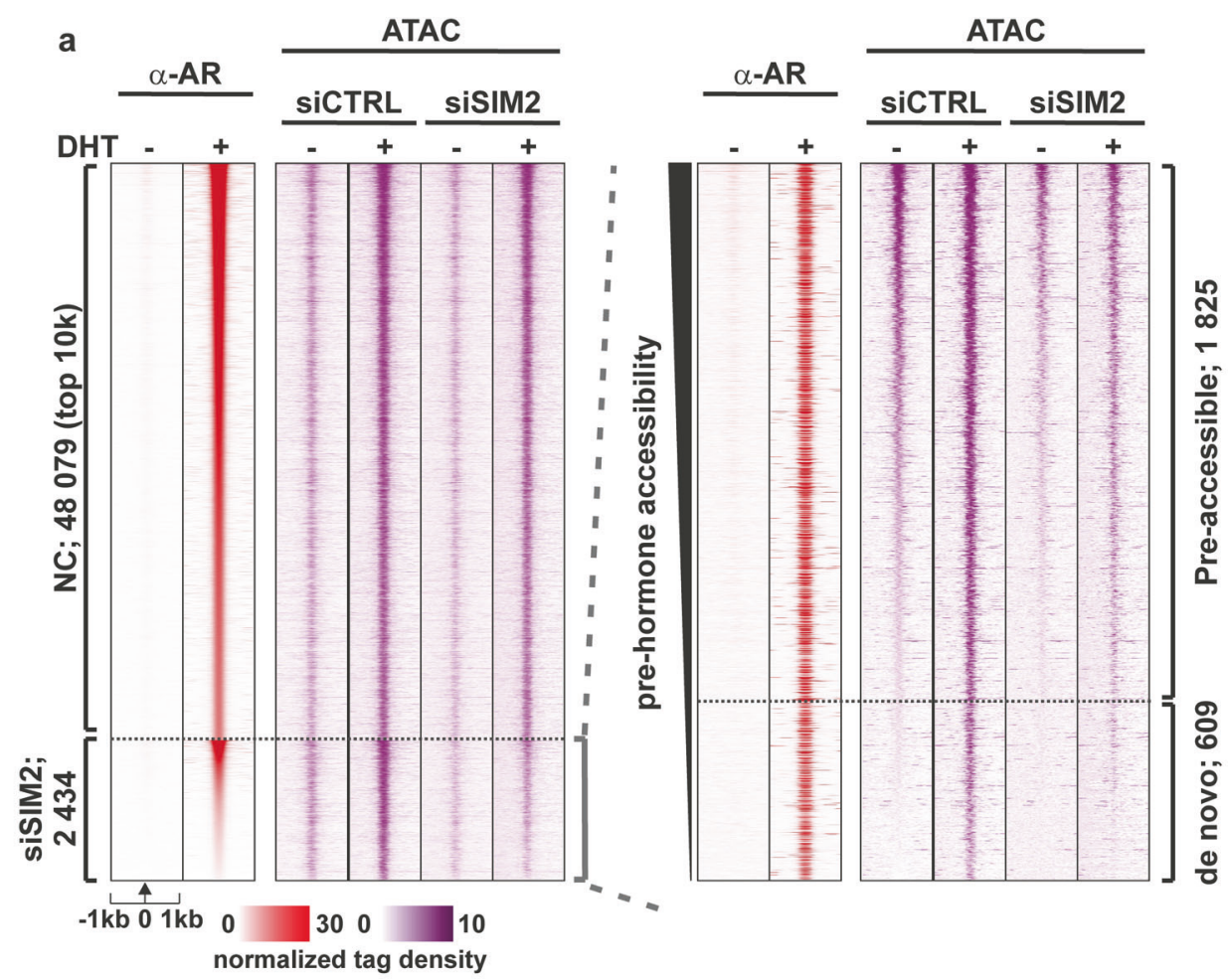

b ATAC c ATAC siSIM2
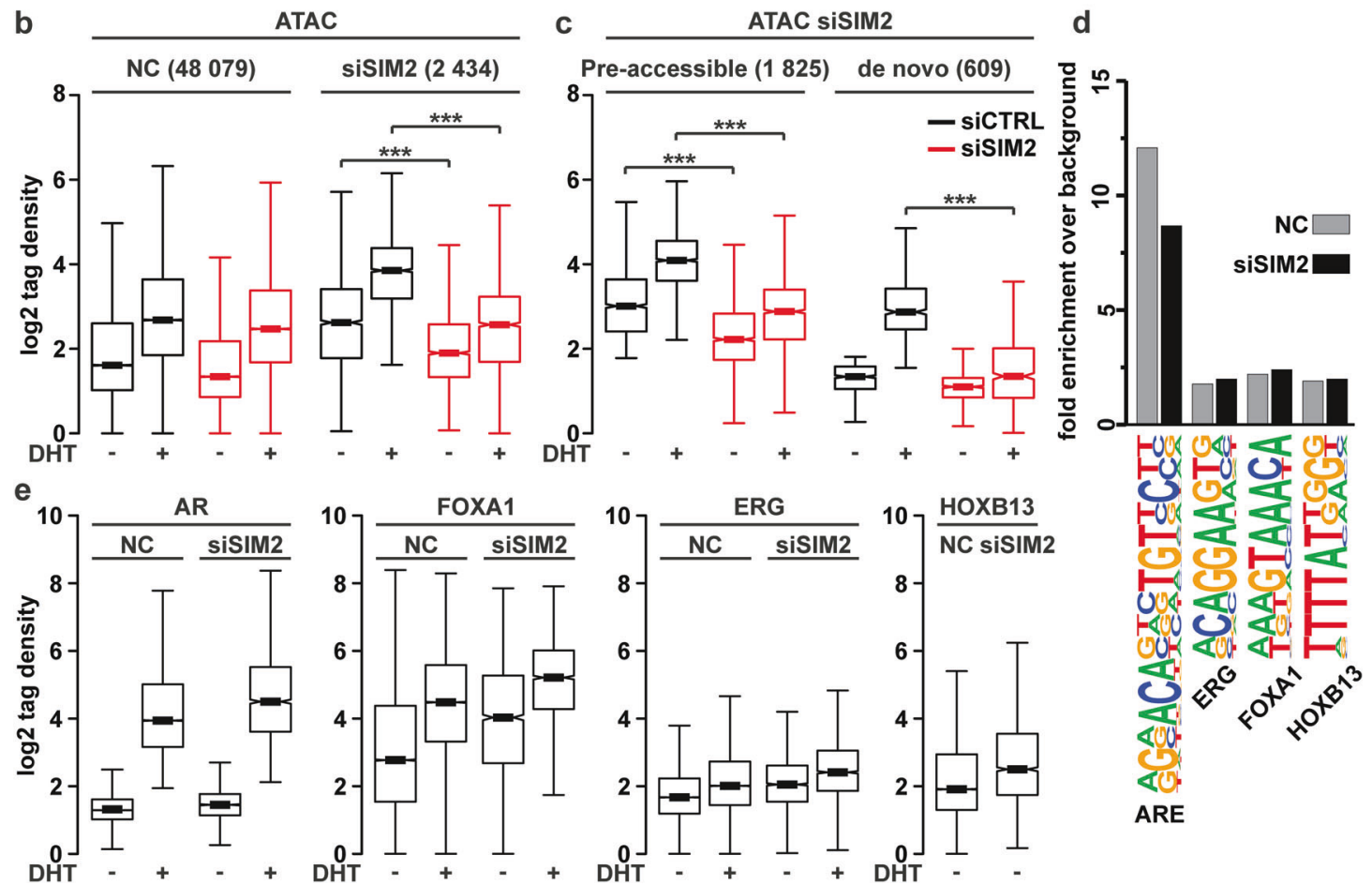

Fig. 6 SIM2 binds to pre-accessible chromatin regions and competes or co-operates with binding of other transcription factors. a Heatmap of normalized tag counts in AR-ChIP-seq and ATAC-seq from siSIM2 and control experiments in VCaP cells with siSIM2-affected chromatin accessibility sites magnified. b Boxplots of ATAC-seq tags in siSIM2-unaffected and -affected chromatin sites (NC and siSIM2, respectively) in the presence and absence of DHT. c ATAC-seq tags at siSIM2-affected pre-accessible and de novo sites. Pre-accessible sites are open already without hormone, whereas de novo sites open with DHT. d Motif analysis of NC and siSIM2-affected sites. e Binding of AR, FOXA1, ERG or HOXB13 at NC and siSIM2-affected sites, respectively in the presence and absence of androgen as indicated. Significant changes in accessibility shown are by asterisks ${ }^{* * *}<0.001$, calculated with One-way ANOVA with Bonferroni post hoc test.

SIM2 silencing affects the cell proliferation. We therefore monitored the effect of SIM2 silencing on VCaP cells with livecell imaging and compared effect of the silencing to that of $A R$ depletion. As assessed by relative cell confluence, SIM2 silencing decelerated VCaP cell growth similarly as that of AR or SMARCA4 (Fig. 5). Proliferation of androgen-exposed LNCaP cells was not however attenuated by SIM2 silencing (Supplementary Fig. S10), but without androgen, SIM2-silenced LNCaP cells interestingly 
a

$\alpha-A R$

$\begin{array}{cc}\frac{\text { ATAC }}{\text { SiCTRL }} & \frac{\text { siSIM2 }}{-}+\frac{1}{4}\end{array}$

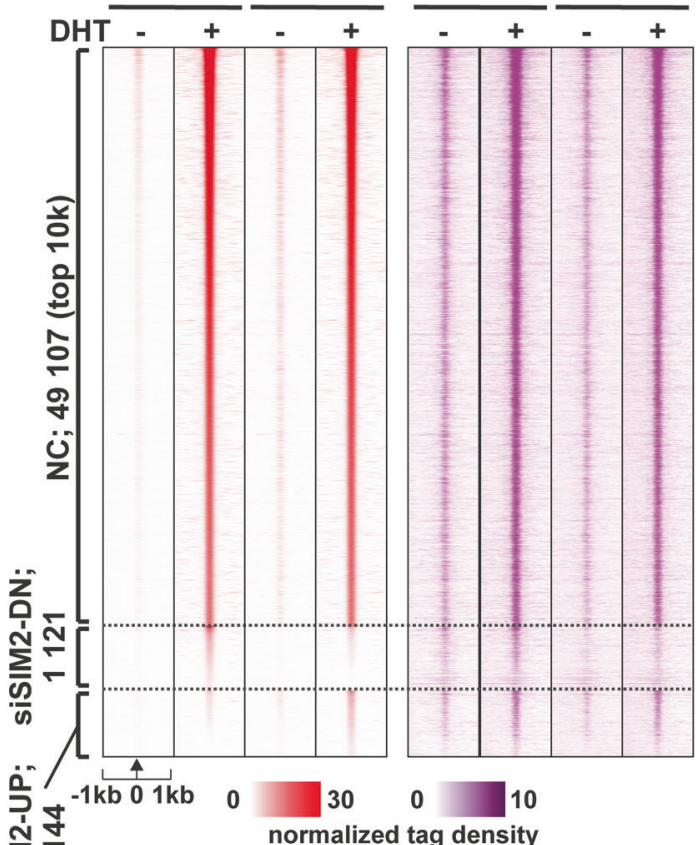

b

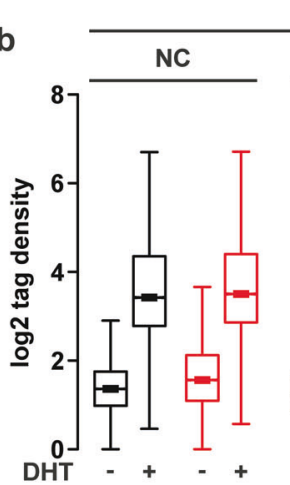

AR
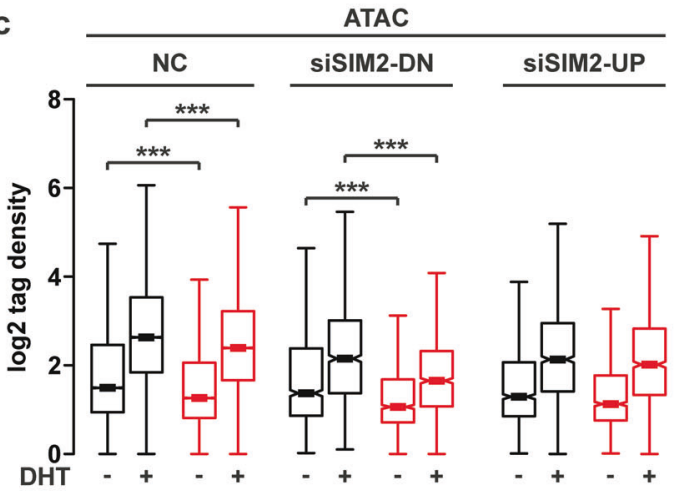

d

fold enrichment over background
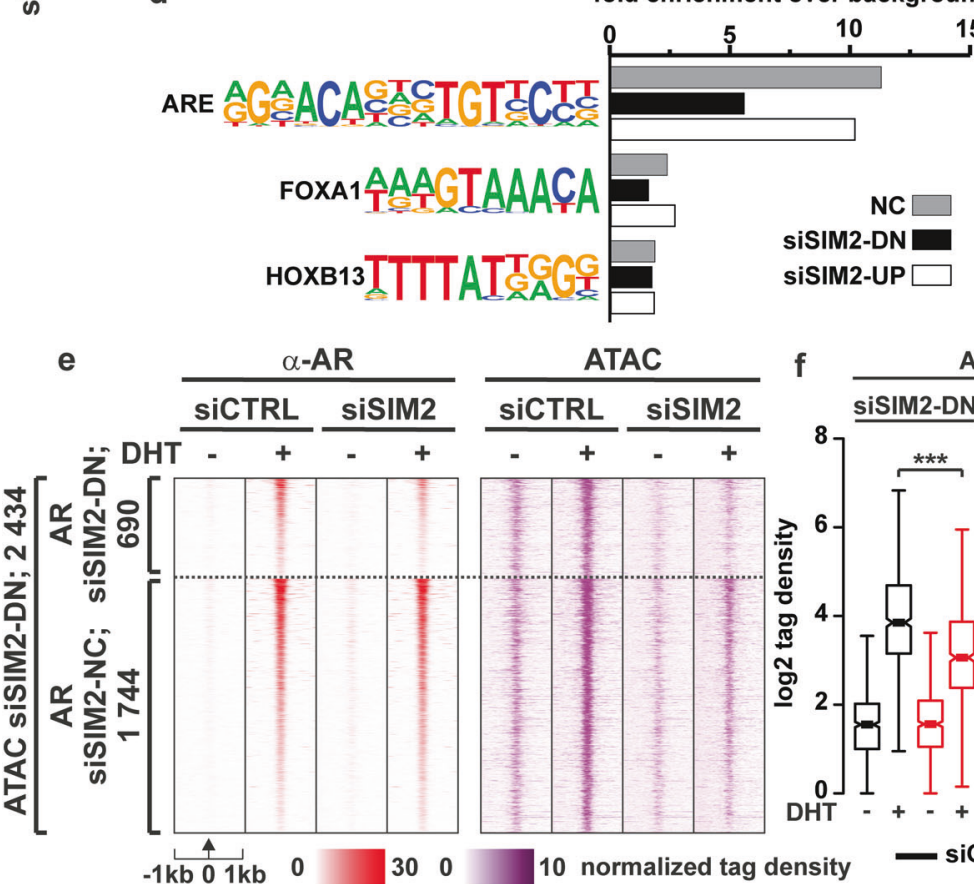

ATAC

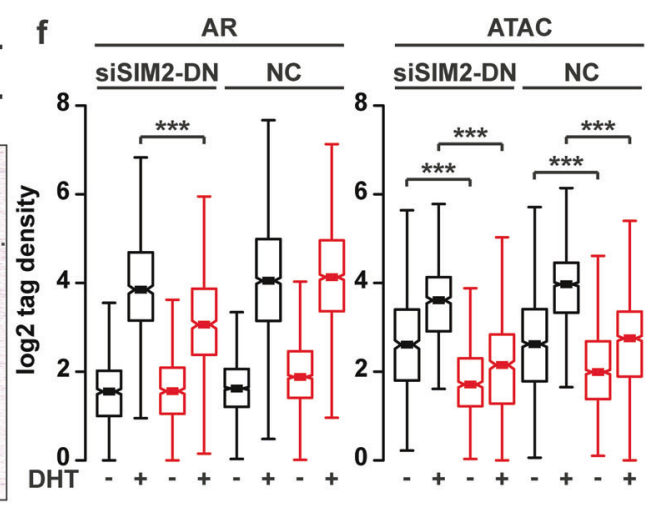

Fig. 7 Silencing of SIM2 alters binding of AR to chromatin at more than two thousand chromatin sites. a AR-ChIP seq and ATAC-seg data shown as heatmap of normalized tag densities upon SIM2 silencing and vehicle or DHT exposure. Sites where AR binding is decreased (siSIM2DN) and increased (siSIM2-UP) by siSIM2 are shown in total and together with top10k of non-changed (NC) sites. Boxplot of AR binding (b) and chromatin accessibility changes (c) in groups defined in panel a. d Motif analyses of each site group in panel a. e ATAC-seq results for siSIM2-affected chromatin accessibility sites divided into 690 SIM2- downregulated AR-binding sites and 1744 nonchanged AR-binding sites. f Boxplots of e. Significance indicated by asterisks ${ }^{* * *}<0.001$ calculated with One-way ANOVA with Bonferroni post hoc test.

proliferated faster than their controls. The reason for these differences between these PCa lines may derive from $\sim 20$-fold higher expression of SIM2 mRNA in VCaP cells than LNCaP cells (Supplementary Fig. S11). To complement the above data with
VCaP cells in three-dimensional tumor growth, we used chick embryo chorioallantoic membrane (CAM) assay (images of representative in ovo tumors in Supplementary Fig. S23a). In line with the results from the cell culture experiments, SIM2 silencing 


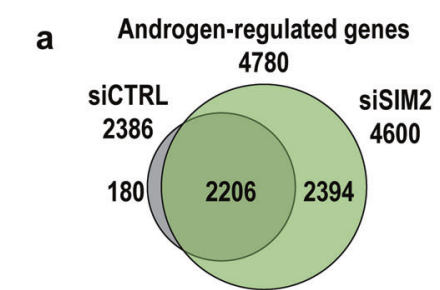

membrane trafficking (152) cellular protein catabolic process (167)

Golgi vesicle transport (94) covalent chromatin modification (105) endomembrane system organization (100)

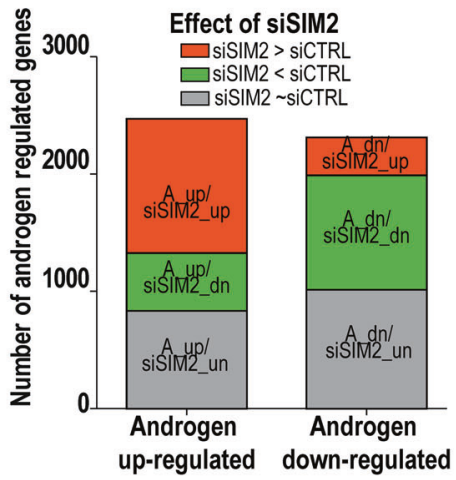

e
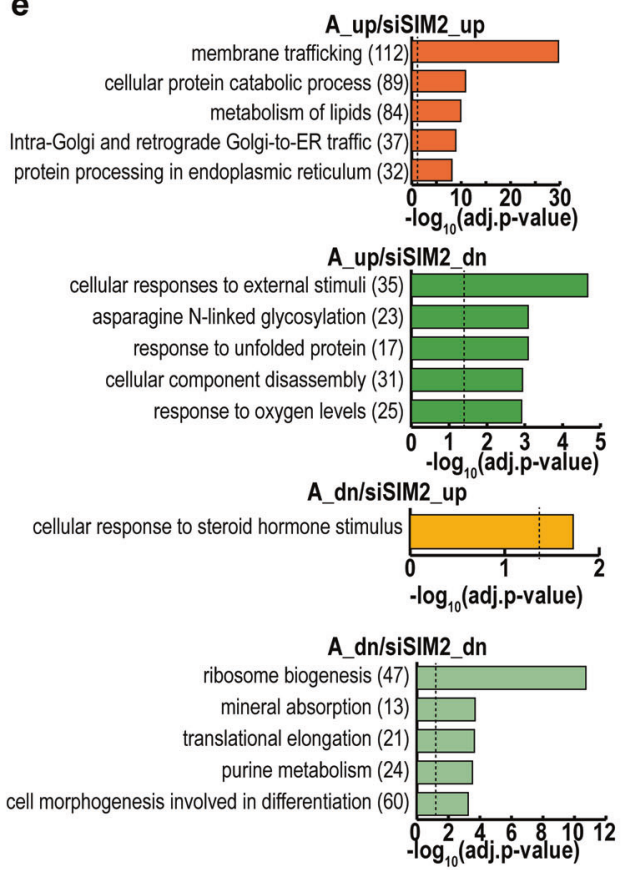

Androgen up-regulated genes

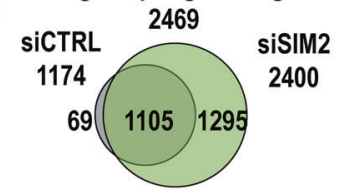

Androgen down-regulated genes
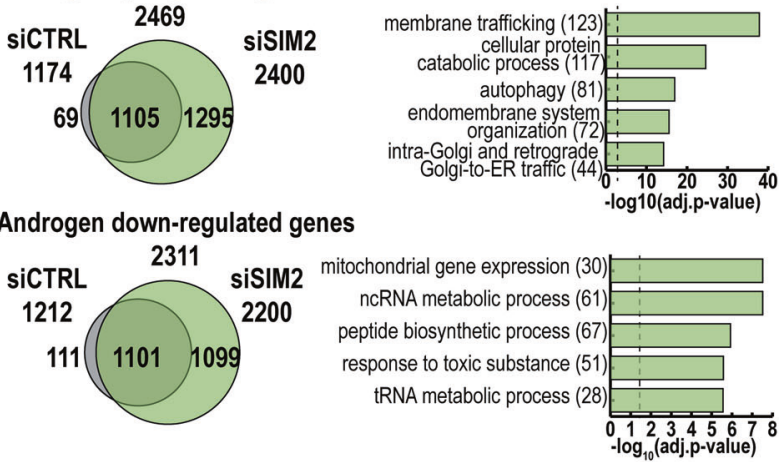

d
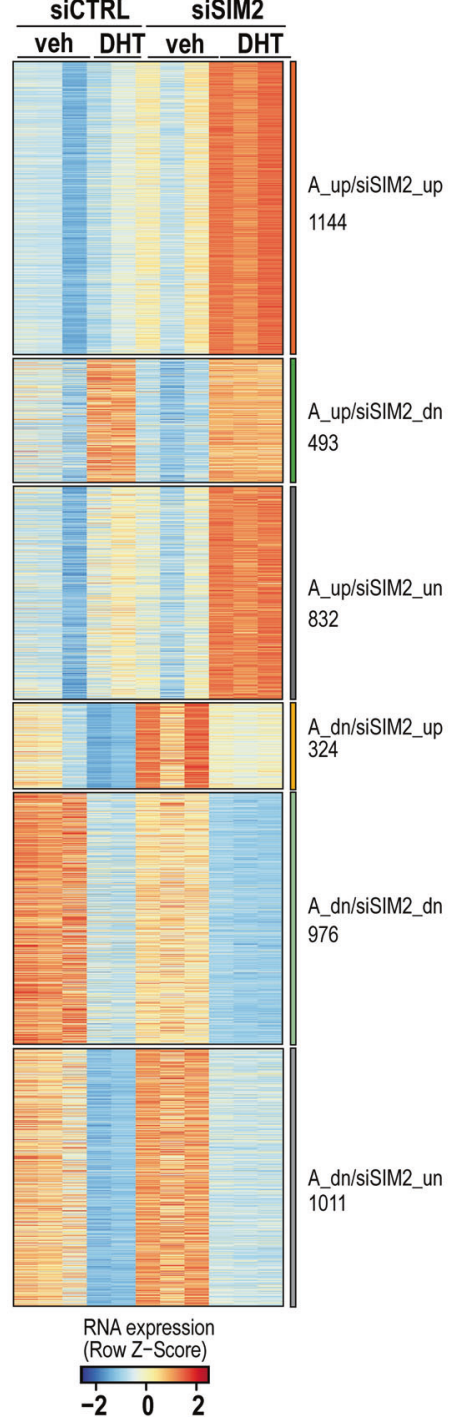

Fig. 8 Silencing of SIM2 in VCaP cells yields nearly three thousand differentially expressed androgen-regulated genes as assessed by RNA-seq. a Venn-diagrams showing the overlap of androgen-regulated genes (adjusted $p$ value $<0.05$ ) in control (siCTRL)- and SIM2-targeting siRNA (siSIM2)-treated cells. Top five enriched pathways for siSIM2 unique androgen-regulated genes are shown in bar plot. b Venn diagrams subdividing androgen-regulated genes into upregulated and downregulated subpopulations with their top five enriched pathways in siSIM2 unique genes in bar plots. c Subdivision of androgen-upregulated and-downregulated genes to six groups as defined by the effect of siSIM2. siSIM2-upregulated genes (A_up/siSIM2_up; A_dn/siSIM2_up; adjusted $p$ value $<0.05, \log 2$ [siSIM2 androgen/siCTRL androgen] $>0$ ) are shown in red, siSIM2-downregulated genes (A_up/siSIM2_dn; A_dn/siSIM2_dn; adjusted $p$ value $<0.05$, log2[siSIM2 androgen/siCTRL androgen] $<0$ ) in green, and unchanged genes (A_up/siSIM2_un; A_dn/siSIM2_un) in gray. $\mathbf{d}$ Heatmap showing RNA expression of individual replicates ( $n=2$ for siCTRL DHT and $n=3$ for others) in the gene groups defined in panel c. Gene numbers belonging to each group are shown on the right. veh, vehicle. e Pathway analysis showing the five most significantly (adjusted $p$ value $<0.05$, dashed line) enriched pathway in Metascape for siSIM2 affected gene sets defined in panel d, see also Supplementary Fig. S13 and Supplementary Table 3. Number of genes associated with each biological process is shown in parenthesis. 
similarly to AR depletion decreased tumor size of VCaP cells in CAM assays (Supplementary Fig. S23b), which further points to the importance of SIM2 in the regulation of CRPC cells.

\section{DISCUSSION}

Here, we utilized ChIP-SICAP which purifies exclusively chromatinbound functional interactors [19] to cast new light to the protein associations and function of AR. Our ChIP-SICAP quantified proteins binding to AR-containing enhancers in PCa cell milieu. Other proteomic approaches that have captured proteins in the proximity of AR on chromatin in PCa cells utilized RIME in CWRR1derived R1-AD1 cells and LNCaP cells $[8,9]$. Although these studies reported similar numbers of proteins as our ChIP-SICAP in VCaP cells, the overlap of identified proteins is small, probably reflecting the intrinsic differences between the methodologies and cell line models. Only three BAF complex members, ARID1A, SMARCA4 and SMARCC1, are identified in all three studies. Both RIME and ChIPSICAP combine ChIP of endogenous protein complexes with MS analysis, however in ChIP-SICAP, ChIP is followed by a purification step to specifically capture DNA-bound proteins $[19,35]$. Therefore, the investigation on chromatin-bound functional interactors of $A R$ through ChIP-SICAP in VCaP cells resulted in a smaller number of identified proteins than RIME in LNCaP cells (190 vs. 333), but a larger portion of the ChIP-SICAP quantified proteins was significantly enriched with AR in androgen-dependent fashion (46\% vs. $20 \%$, respectively). VCaP cells, but not $\mathrm{LNCaP}$ cells, additionally express an AR variant, AR-V7, devoid of the ligand-binding domain (LBD) [36]. Moreover, the VCaP cells, but not the LNCaP cells, express a TMPRSS2-ERG fusion, and the AR-LBD in LNCaP is pointmutated. These differences may contribute to the low overlap between the AR chromatin protein interactomes of the VCaP and the LNCaP cells, but they are not likely to explain all of them.

SMARCA4 is an established chromatin remodeler [37] and one of the shared members of AR chromatomes identified in three studied PCa cell lines $[8,9]$. It is overexpressed in TCGA PCa data [38] and PCa cell lines [39]. Higher SMARCA4 expression correlates with aggressiveness of the disease, but irrespective of clinical or molecular subtype $[12,13,39,40]$. SMARCA4 and PTEN (frequently lost in PCa) are interestingly synthetic lethal in PCa [13], which is reflected in PCa cell models as an altered chromatin structure driving a protumorigenic transcriptome [13].

To the best of our knowledge, the impact of androgen-activated AR on the chromatin occupancy of SMARCA4, the chromatin accessibility and the AR target gene expression has not previously been investigated in an integrative fashion in PCa cells. Our data from VCaP cells show that despite a marked number of SMARCA4 chromatin-binding sites responded to androgen (>40\%) and a large overlap (75\%) between SMARCA4- and AR-binding sites, the depletion of SMARCA4 changed the chromatin accessibility $<10 \%$ of those sites. This relatively small effect of SMARCA4 depletion may be explained by two interchangeable ATPase subunits, SMARCA4 and SMARCA2, the latter of which was not however detected in our AR ChIP-SICAP. Moreover, our data did not indicate any compensatory increase in the expression of SMARCA2 upon SMARCA4 depletion. Since enhancers recruit remodelers with different affinity [41], removal of SMARCA4 and BAF activity could have paved the way to another remodeling activity to the enhancer. The NuRD complex whose subunits, MTA1, GATA2B and CHD4 (ATPase subunit), identified here could represent an alternative remodeling activity. In fact, many enhancers require the activity of two or more remodeling complexes, e.g. SMARCA4 and CHD4-containing ones [41].

Regardless of the relatively limited effect of SMARCA4 on the chromatin accessibility on ARBs, SMARCA4 depletion affected chromatin accessibility at a larger number of sites ( 11000). The depletion did not only decrease the accessibility, but the accessibility at $\sim 20 \%$ of the SMARCA4 depletion-affected sites was increased, with FOXA1 being enriched at decreased sites. The FOXA1 has been assumed to create open chromatin environment by displacing linker histones $[42,43]$. Our results suggest that BAF complexes might have more prevalent roles in the FOXA1-mediated regulation of chromatin accessibility than previously postulated.

The SMARCA4 depletion in VCaP cells had interesting effects on AR-mediated gene expression, as e.g. the expression of androgenregulated genes involved in the extracellular matrix organization and morphogenesis of branching structures were sensitive to SMARCA4. These pathways include possible connections to EMT that has previously been indicated as a cellular process regulated by SMARCA4 [44-49]. Similarly to our study, larger and more general effects in chromatin accessibility than those in gene expression were demonstrated by SMARCA4 in other cancer cell models $[50,51]$. We found that SMARCA4 depletion practically blunted the proliferation-promoting effect of androgen in VCaP cells, which is accordance with others' studies with LNCaP cells in which the effect of androgen was not however addressed [39, 40].

Our chromatin-directed proteomics approach revealed SIM2, a basic helix-loop-helix Per-Arnt-Sim (bHLH-PAS) TF [52], and its heterodimerization partner ARNT as members of AR chromatome. The bHLH-PAS TFs play important roles in morphogenesis and controlling circadian rhythmicity, responses to hypoxia and toxin metabolism [53]. The SIM2-ARNT heterodimer can either activate or repress transcription depending on the gene context $[52,54,55]$. This notion is in line with our genome-wide data from PCa cells, showing that silencing of SIM2 influenced activated and repressed - similar numbers of androgenregulated target genes. For example, the expression of genes enriched in cellular response to steroid hormone stimulus were enhanced by SIM2 silencing, whereas the expression of genes enriched in ribosome biogenesis were repressed by the silencing. Additionally, SIM2 silencing showed androgenindependent effects on genes involved in cell cycle process, cell division and DNA repair; e.g. enhancing the expression of $B R C A 1, B R C A 2$ and ATM. These roles are in line with SIM2's growth-promoting role [56-58], ability to regulate DNA damage repair $[59,60]$ and increase invasion potential [61]. Moreover, we show that the proliferation of VCaP cells and their tumor size are affected by SIM2 expression. Since the SIM2 is overexpressed in $\mathrm{PCa}$, with the expression increasing with the aggressiveness of $\mathrm{PCa}[21,22]$, these data point to the relevance and importance of SIM2 as a PCa biomarker. Moreover, given the role of SIM2 in neurogenesis $[62,63]$ and that the expression of neuroendocrine markers SYP, CHGA and NSE $[64,65]$ are attenuated by SIM2 silencing, the SIM2 may also have a role in the neuroendocrine $P C a$.

Our ATAC-seq showed that, although most of the ARBs were unaffected by SIM2 silencing, the accessibility at $>2000$ ARBs was altered. Chromatin at most of the affected sites was accessible before androgen exposure, suggesting that SIM2 preferentially binds to and acts at open chromatin regions. In addition, binding of AR was affected - attenuated or enhanced - at 2000 sites by SIM2 silencing. However, there was no simple relationship between the changes in chromatin accessibility and the AR binding upon SIM2 silencing. SIM2 could display some pioneer factor properties with AR in certain chromatin environments, but these properties are much weaker than those of the FOXA1. Moreover, the majority of ARBs affected by SIM2 silencing do not overlap with those altered by FOXA1 depletion. Together, our results indicate that the SIM2 is a TF co-operating with AR in CRPC cells.

In conclusion, our results confirm the chromatin opening role of SMARCA4 in AR-mediated gene regulation, which is interestingly reflected in the expression of genes involved in pathways potentially connected with EMT in PCa. In addition, our results indicate that SIM2 plays an important, AR target pathway-selective role in the regulation of CRPC cells. Finally, the chromatome of AR 
in CRPC cells identified herein forms an important resource for the AR field, focusing on this important drug target.

\section{MATERIALS AND METHODS \\ Cell culture}

VCaP cells were obtained from ATCC, tested negative for mycoplasma, and verified by Institute for Molecular Medicine Finland. Cells were maintained in DMEM (Gibco \#41965-039) supplemented with 10\% FBS (HyClone \#AYE161472) and $1 \mathrm{U} / \mu \mathrm{l}$ penicillin and $1 \mu \mathrm{g} / \mathrm{ml}$ streptomycin (Gibco $\# 15140-122)$

\section{ChIP-SICAP}

SILAC-labeled VCaP cells $\left(9 \times 10^{6}\right.$ cells/ $15-\mathrm{cm}$ culture dish, Supplementary materials) were cultured for $72 \mathrm{~h}$, before adding $10 \mathrm{nM}$ R1881 to heavylabeled (Arg10, Lys8) cells and ethanol (vehicle) to light-labeled (Arg0, Lys0) cells for $1 \mathrm{~h}$. Cells were immunoprecipitated as in [66] with following modifications; the chromatin was incubated overnight with aAR [67] followed by $3 \mathrm{~h}$ incubation with rotating $\mathrm{G}$ protein-coupled DynaBeads (Invitrogen) at $4{ }^{\circ} \mathrm{C}$. ChIP protocol was subsequently followed by [19, 35]. (Details and data analysis in Supplementary materials).

\section{RNAi followed by RNA-sequencing}

For RNA-sequencing, VCaP cells $\left(0.7 \times 10^{6}\right.$ cells/well of 6-well plate) were reverse-transfected with ON-TARGETplus SMARTpools for SMARCA4, SIM2 and non-targeting control (Dharmacon, Supplementary materials) using RNAiMAX (ThermoFisher Scientific) according to the manufacturer's instructions (Details in Supplementary materials).

\section{ChIP- and ATAC-sequencing}

VCaP cells $\left(5 \times 10^{6}\right.$ cells $/ 10$-cm culture dish) were cultured in maintenance medium for $48 \mathrm{~h}$. Medium was changed to DMEM, $2.5 \%$ charcoal-stripped FBS for $48 \mathrm{~h}$ prior to $1 \mathrm{~h}$ exposure with DHT $100 \mathrm{nM}$ or vehicle. In siRNAexperiments, SMARCA4, SIM2 or FOXA1 were silenced by reverse transfection with ON-TARGETplus SMARTpools for $96 \mathrm{~h}$ prior hormone treatment. ChIPs were performed as in [66] with aSMARCA4 (Abcam, \#ab110641), aFOXA1 (Abcam, \#ab23738) and aAR [67]. The libraries were prepared with NEB Next ${ }^{\circ}$ Ultra DNA ${ }^{T M}$ II kit $(E 7103, N E B)$ and sequenced with Illumina NextSeq 500 (75SE). (Details and data analysis in Supplementary materials).

\section{DATA AVAILABILITY}

The MS data have been deposited to the ProteomeXchange Consortium via the PRIDE [79] partner repository with the dataset identifier PXD025193. ATAC-seq, ChIPseq and RNA-seq datasets have been submitted to GEO database with accession code: GSE136016.

\section{REFERENCES}

1. Wong YN, Ferraldeschi R, Attard G, de Bono J. Evolution of androgen receptor targeted therapy for advanced prostate cancer. Nat Rev Clin Oncol. 2014;11:365-76.

2. Rajaram P, Rivera A, Muthima K, Olveda N, Muchalski H, Chen QH. Secondgeneration androgen receptor antagonists as hormonal therapeutics for three forms of prostate cancer. Molecules. 2020. https://doi.org/10.3390/molecules25102448.

3. Agoulnik IU, Weigel NL. Coactivator selective regulation of androgen receptor activity. Steroids. 2009;74:669-74.

4. Heemers HV, Tindall DJ. Androgen receptor (AR) coregulators: a diversity of functions converging on and regulating the AR transcriptional complex. Endocr Rev. 2007;28:778-808.

5. Heemers HV, Regan KM, Schmidt LJ, Anderson SK, Ballman KV, Tindall DJ. Androgen modulation of coregulator expression in prostate cancer cells. Mol Endocrinol. 2009;23:572-83.

6. Lambert JP, Pawson T, Gingras AC. Mapping physical interactions within chromatin by proteomic approaches. Proteomics. 2012;12:1609-22.

7. Lambert JP, Tucholska M, Pawson T, Gingras AC. Incorporating DNA shearing in standard affinity purification allows simultaneous identification of both soluble and chromatin-bound interaction partners. J Proteom. 2014;100:55-59.

8. Paltoglou S, Das R, Townley SL, Hickey TE, Tarulli GA, Coutinho I, et al. Novel androgen receptor coregulator GRHL2 exerts both oncogenic and antimetastatic functions in prostate cancer. Cancer Res. 2017;77:3417-30.
9. Stelloo S, Nevedomskaya E, Kim Y, Hoekman L, Bleijerveld OB, Mirza T, et al. Endogenous androgen receptor proteomic profiling reveals genomic subcomplex involved in prostate tumorigenesis. Oncogene. 2018;37:313-22.

10. Roesley SNA, La Marca JE, Deans AJ, Mckenzie L, Suryadinata R, Burke $P$, et al. Phosphorylation of Drosophila Brahma on CDK-phosphorylation sites is important for cell cycle regulation and differentiation. Cell Cycle. 2018;17:1559-78.

11. Wang G, Zhao D, Spring DJ, DePinho RA. Genetics and biology of prostate cancer. Genes Dev. 2018;32:1105-40.

12. Muthuswami R, Bailey L, Rakesh R, Imbalzano AN, Nickerson JA, Hockensmith JW. BRG1 is a prognostic indicator and a potential therapeutic target for prostate cancer. J Cell Physiol. 2019.

13. Ding Y, Li N, Dong B, Guo W, Wei H, Chen Q, et al. Chromatin remodeling ATPase BRG1 and PTEN are synthetic lethal in prostate cancer. J Clin Invest. 2019;129:759-73.

14. Centenera MM, Selth LA, Ebrahimie E, Butler LM, Tilley WD. New opportunities for targeting the androgen receptor in prostate cancer. Cold Spring Harb Perspect Med. 2018. https://doi.org/10.1101/cshperspect.a030478.

15. Sandoval GJ, Pulice JL, Pakula H, Schenone M, Takeda DY, Pop M, et al. Binding of TMPRSS2-ERG to BAF Chromatin Remodeling Complexes Mediates Prostate Oncogenesis. Mol Cell. 2018;71:554-66. e7

16. Hankey W, Chen Z, Wang Q. Shaping chromatin states in prostate cancer by pioneer transcription factors. Cancer Res. 2020;80:2427-36.

17. Sahu B, Laakso M, Ovaska K, Mirtti T, Lundin J, Rannikko A, et al. Dual role of FoxA1 in androgen receptor binding to chromatin, androgen signalling and prostate cancer. EMBO J. 2011;30:3962-76.

18. Pomerantz MM, Li F, Takeda DY, Lenci R, Chonkar A, Chabot M, et al. The androgen receptor cistrome is extensively reprogrammed in human prostate tumorigenesis. Nat Genet. 2015;47:1346-51.

19. Rafiee MR, Girardot C, Sigismondo G, Krijgsveld J. Expanding the circuitry of pluripotency by selective isolation of chromatin-associated proteins. Mol Cell. 2016;64:624-35.

20. Labrecque MP, Coleman IM, Brown LG, True LD, Kollath L, Lakely B, et al. Molecular profiling stratifies diverse phenotypes of treatment-refractory metastatic castration-resistant prostate cancer. J Clin Invest. 2019;130:4492-505.

21. Halvorsen OJ, Rostad K, Oyan AM, Puntervoll H, Bo TH, Stordrange $L$, et al. Increased expression of SIM2-s protein is a novel marker of aggressive prostate cancer. Clin Cancer Res. 2007;13:892-7.

22. Lu B, Asara JM, Sanda MG, Arredouani MS. The role of the transcription factor SIM2 in prostate cancer. PLoS One. 2011;6:e28837.

23. Lempiainen JK, Niskanen EA, Vuoti KM, Lampinen RE, Goos $H$, Varjosalo $M$, et al. Agonist-specific Protein Interactomes of Glucocorticoid and Androgen Receptor as Revealed by Proximity Mapping. Mol Cell Proteom. 2017;16:1462-74.

24. O'Rourke DJ, DiJohnson DA, Caiazzo RJ Jr, Nelson JC, Ure D, O'Leary MP, et al. Autoantibody signatures as biomarkers to distinguish prostate cancer from benign prostatic hyperplasia in patients with increased serum prostate specific antigen. Clin Chim Acta. 2012;413:561-7.

25. Xu L, Lee JR, Hao S, Ling XB, Brooks JD, Wang SX, et al. Improved detection of prostate cancer using a magneto-nanosensor assay for serum circulating autoantibodies. PLoS One. 2019;14:e0221051.

26. Sharma A, Mendonca J, Ying J, Kim HS, Verdone JE, Zarif JC, et al. The prostate metastasis suppressor gene NDRG1 differentially regulates cell motility and invasion. Mol Oncol. 2017;11:655-69.

27. Toropainen S, Malinen M, Kaikkonen S, Rytinki M, Jaaskelainen $T$, Sahu B, et al. SUMO ligase PIAS1 functions as a target gene selective androgen receptor coregulator on prostate cancer cell chromatin. Nucleic Acids Res. 2015;43:848-61.

28. Sharma NL, Massie CE, Butter F, Mann M, Bon H, Ramos-Montoya A, et al. The ETS family member GABPalpha modulates androgen receptor signalling and mediates an aggressive phenotype in prostate cancer. Nucleic Acids Res. 2014;42:6256-69.

29. Kron KJ, Murison A, Zhou S, Huang V, Yamaguchi TN, Shiah YJ, et al. TMPRSS2ERG fusion co-opts master transcription factors and activates NOTCH signaling in primary prostate cancer. Nat Genet. 2017;49:1336-45.

30. Paakinaho V, Swinstead EE, Presman DM, Grontved L, Hager GL. Meta-analysis of chromatin programming by steroid receptors. Cell Rep. 2019;28:3523-34. e2

31. Zhou Y, Zhou B, Pache L, Chang M, Khodabakhshi AH, Tanaseichuk O, et al. Metascape provides a biologist-oriented resource for the analysis of systemslevel datasets. Nat Commun. 2019;10:1523-019-09234-6.

32. Arredouani MS, Lu B, Bhasin M, Eljanne M, Yue W, Mosquera JM, et al. Identification of the transcription factor single-minded homologue 2 as a potential biomarker and immunotherapy target in prostate cancer. Clin Cancer Res. 2009;15:5794-802.

33. Annala M, Kivinummi K, Tuominen J, Karakurt S, Granberg K, Latonen L, et al. Recurrent SKIL-activating rearrangements in ETS-negative prostate cancer. Oncotarget. 2015;6:6235-50. 
34. Qin Q, Fan J, Zheng R, Wan C, Mei S, Wu Q, et al. Lisa: inferring transcriptional regulators through integrative modeling of public chromatin accessibility and ChIP-seq data. Genome Biol. 2020;21:32-020-1934-6.

35. Rafiee MR, Sigismondo G, Kalxdorf M, Forster L, Brugger B, Bethune J, et al. Proteaseresistant streptavidin for interaction proteomics. Mol Syst Biol. 2020;16:e9370.

36. Yu Z, Chen S, Sowalsky AG, Voznesensky OS, Mostaghel EA, Nelson PS, et al. Rapid induction of androgen receptor splice variants by androgen deprivation in prostate cancer. Clin Cancer Res. 2014;20:1590-1600.

37. Roberts CW, Orkin SH. The SWI/SNF complex-chromatin and cancer. Nat Rev Cancer. 2004;4:133-42.

38. Cancer Genome Atlas Research Network. The molecular taxonomy of primary prostate cancer. Cell. 2015;163:1011-25.

39. Giles KA, Gould CM, Achinger-Kawecka J, Page SG, Kafer GR, Rogers S, et al. BRG1 knockdown inhibits proliferation through multiple cellular pathways in prostate cancer. Clin Epigenetics. 2021;13:37-021-01023-7.

40. Cyrta J, Augspach A, De Filippo MR, Prandi D, Thienger P, Benelli M, et al. Role of specialized composition of SWI/SNF complexes in prostate cancer lineage plasticity. Nat Commun. 2020;11:5549-020-19328-1.

41. Morris SA, Baek S, Sung MH, John S, Wiench M, Johnson TA, et al. Overlapping chromatin-remodeling systems collaborate genome wide at dynamic chromatin transitions. Nat Struct Mol Biol. 2014;21:73-81.

42. Zaret KS, Carroll JS. Pioneer transcription factors: establishing competence for gene expression. Genes Dev. 2011;25:2227-41.

43. Swinstead EE, Paakinaho V, Presman DM, Hager GL. Pioneer factors and ATP dependent chromatin remodeling factors interact dynamically: A new perspective: Multiple transcription factors can effect chromatin pioneer functions through dynamic interactions with ATP-dependent chromatin remodeling factors. Bioessays. 2016;38:1150-7.

44. Matsubara D, Kishaba Y, Ishikawa S, Sakatani T, Oguni S, Tamura T, et al. Lung cancer with loss of BRG1/BRM, shows epithelial mesenchymal transition phenotype and distinct histologic and genetic features. Cancer Sci. 2013;104:266-73.

45. Zhou Z, Su Y, Fa X. Restoration of BRG1 inhibits proliferation and metastasis of lung cancer by regulating tumor suppressor miR-148b. Onco Targets Ther. 2015;8:3603-12.

46. Roy N, Malik S, Villanueva KE, Urano A, Lu X, Von Figura G, et al. Brg1 promotes both tumor-suppressive and oncogenic activities at distinct stages of pancreatic cancer formation. Genes Dev. 2015;29:658-71.

47. Yang $Y$, Liu L, Li M, Cheng X, Fang $M$, Zeng $Q$, et al. The chromatin remodeling protein BRG1 links ELOVL3 trans-activation to prostate cancer metastasis. Biochim Biophys Acta Gene Regul Mech. 2019;1862:834-45.

48. Lv DJ, Song XL, Huang B, Yu YZ, Shu FP, Wang C, et al. HMGB1 Promotes Prostate Cancer Development and Metastasis by Interacting with Brahma-Related Gene 1 and Activating the Akt Signaling Pathway. Theranostics. 2019;9:5166-82.

49. Ailiken G, Kitamura K, Hoshino T, Satoh M, Tanaka N, Minamoto T, et al. Posttranscriptional regulation of BRG1 by FIRDeltaexon2 in gastric cancer. Oncogenesis 2020;9:26-020-0205-4.

50. Schick S, Rendeiro AF, Runggatscher K, Ringler A, Boidol B, Hinkel M, et al. Systematic characterization of BAF mutations provides insights into intracomplex synthetic lethalities in human cancers. Nat Genet. 2019;51:1399-410.

51. Lazar JE, Stehling-Sun S, Nandakumar V, Wang H, Chee Daniel,R, Chee DR, et al. Global regulatory DNA potentiation by SMARCA4 propagates to selective gene expression programs via domain-level remodeling. Cell Rep. 2020;31:107788.

52. Woods SL, Whitelaw ML. Differential activities of murine single minded 1 (SIM1) and SIM2 on a hypoxic response element. Cross-talk between basic helix-loop-helix/perArnt-Sim homology transcription factors. J Biol Chem. 2002;277:10236-43.

53. Wu D, Rastinejad F. Structural characterization of mammalian bHLH-PAS transcription factors. Curr Opin Struct Biol. 2017:43(Apr):1-9.

54. Metz RP, Kwak HI, Gustafson T, Laffin B, Porter WW. Differential transcriptional regulation by mouse single-minded 2s. J Biol Chem. 2006;281:10839-48.

55. Woods S, Farrall A, Procko C, Whitelaw ML. The bHLH/Per-Arnt-Sim transcription factor SIM2 regulates muscle transcript myomesin2 via a novel, non-canonical Ebox sequence. Nucleic Acids Res. 2008;36(Jun):3716-27.

56. Aleman MJ, DeYoung MP, Tress M, Keating P, Perry GW, Narayanan R. Inhibition of single minded 2 gene expression mediates tumor-selective apoptosis and differentiation in human colon cancer cells. Proc Natl Acad Sci USA. 2005;102:12765-70.

57. DeYoung MP, Tress M, Narayanan R. Down's syndrome-associated Single Minded 2 gene as a pancreatic cancer drug therapy target. Cancer Lett. 2003;200:25-31.

58. DeYoung MP, Tress M, Narayanan R. Identification of Down's syndrome critical locus gene SIM2-s as a drug therapy target for solid tumors. Proc Natl Acad Sci USA. 2003;100:4760-5.

59. Kwak HI, Gustafson T, Metz RP, Laffin B, Schedin P, Porter WW. Inhibition of breast cancer growth and invasion by single-minded 2s. Carcinogenesis. 2007; 28:259-66.
60. Pearson SJ, Roy Sarkar T, McQueen CM, Elswood J, Schmitt EE, Wall SW, et al. ATM-dependent activation of SIM2s regulates homologous recombination and epithelial-mesenchymal transition. Oncogene. 2019;38:2611-26.

61. He Q, Li G, Su Y, Shen J, Liu Q, Ma X, et al. Single minded 2-s (SIM2-s) gene is expressed in human GBM cells and involved in GBM invasion. Cancer Biol Ther. 2010;9:430-6.

62. Dahmane N, Charron G, Lopes C, Yaspo ML, Maunoury C, Decorte L, et al. Down syndrome-critical region contains a gene homologous to Drosophila sim expressed during rat and human central nervous system development. Proc Natl Acad Sci USA. 1995;92:9191-5.

63. Rachidi M, Lopes C, Charron G, Delezoide AL, Paly E, Bloch B, et al. Spatial and temporal localization during embryonic and fetal human development of the transcription factor SIM2 in brain regions altered in Down syndrome. Int J Dev Neurosci. 2005;23:475-84.

64. Fine SW. Neuroendocrine tumors of the prostate. Mod Pathol. 2018;31: S122-132.

65. Hu J, Han B, Huang J. Morphologic spectrum of neuroendocrine tumors of the prostate: an updated review. Arch Pathol Lab Med. 2020;144:320-5.

66. Paakinaho V, Kaikkonen S, Makkonen H, Benes V, Palvimo JJ. SUMOylation regulates the chromatin occupancy and anti-proliferative gene programs of glucocorticoid receptor. Nucleic Acids Res. 2014;42:1575-92.

67. Karvonen U, Kallio PJ, Janne OA, Palvimo JJ. Interaction of androgen receptors with androgen response element in intact cells. Roles of amino- and carboxylterminal regions and the ligand. J Biol Chem. 1997;272:15973-9.

\section{ACKNOWLEDGEMENTS}

Merja Räsänen, Eija Korhonen, Aija Kekkonen, and Kaisa Luostari are acknowledged for their expert technical assistance. The EMBL GeneCore is acknowledged for deep sequencing, the UEF Bioinformatics Center for providing computational infrastructure and the UEF Cell and Tissue Imaging Unit for supporting live-cell imaging experiments.

\section{FUNDING}

G.S. and J.K. acknowledge the Excellence Cluster CellNetworks (EXC81). This paper was supported by UEF DPMM, Academy of Finland, Sigrid Jusélius Foundation, Cancer Foundation Finland, and Paavo Koistinen Foundation. Open access funding provided by University of Eastern Finland (UEF) including Kuopio University Hospital.

\section{COMPETING INTERESTS}

The authors declare no competing interest.

\section{ADDITIONAL INFORMATION}

Supplementary information The online version contains supplementary material available at https://doi.org/10.1038/s41388-021-01887-2.

Correspondence and requests for materials should be addressed to J.J.P.

Reprints and permission information is available at http://www.nature.com/ reprints

Publisher's note Springer Nature remains neutral with regard to jurisdictional claims in published maps and institutional affiliations. Attribution 4.0 International License, which permits use, sharing, adaptation, distribution and reproduction in any medium or format, as long as you give appropriate credit to the original author(s) and the source, provide a link to the Creative Commons license, and indicate if changes were made. The images or other third party material in this article are included in the article's Creative Commons license, unless indicated otherwise in a credit line to the material. If material is not included in the article's Creative Commons license and your intended use is not permitted by statutory regulation or exceeds the permitted use, you will need to obtain permission directly from the copyright holder. To view a copy of this license, visit http://creativecommons. org/licenses/by/4.0/.

(c) The Author(s) 2021 